\title{
Nonequilibrium Phase Transition for a Heavy Particle in a Granular Fluid
}

\author{
Andrés Santost and James W. Dufty \\ Department of Physics, University of Florida, Gainesville, FL 32611
}

(November 2, 2018)

PACS number(s): 45.70.Mg, 05.20.Dd, 05.40.Jc

\begin{abstract}
It is shown that the homogeneous cooling state (HCS) for a heavy impurity particle in a granular fluid supports two distinct phases. The order parameter $\bar{\phi}_{\mathrm{s}}$ is the mean square velocity of the impurity particle relative to that of a fluid particle, and the control parameter $\xi^{*}$ is the fluid cooling rate relative to the impurity collision rate. For $\xi^{*}<1$ there is a "normal" phase for which $\bar{\phi}_{\mathrm{s}}$ scales as the fluid/impurity mass ratio, just as for a system with elastic collisions. For $\xi^{*}>1$ an "ordered" phase occurs in which $\bar{\phi}_{\mathrm{s}}$ is finite even for vanishingly small mass ratio, representing an extreme violation of energy equipartition. The phenomenon can be described in terms of a Landaulike free energy for a second order phase transition. The dynamics leading to the HCS is studied in detail using an asymptotic analysis of the Enskog-Lorentz kinetic equation near each phase and the critical domain. Critical slowing is observed with a divergent relaxation time at the critical point. The stationary velocity distributions are determined in each case, showing a crossover from Maxwellian in the normal phase to an exponential quartic function of the velocity that is sharply peaked about the non-zero $\bar{\phi}_{\mathrm{s}}$ for the ordered phase. It is shown that the diffusion coefficient in the normal phase diverges at the critical point and remains so in the ordered phase. This is interpreted as a transition from diffusive to ballistic dynamics between the normal and ordered phases.
\end{abstract}

\section{INTRODUCTION}

A mixture of two mechanically different fluids rapidly approaches a common equilibrium state for times larger than a mean free time. This equilibrium state is characterized by a common temperature or, equivalently, mean square velocities for each type of particle that differ by their mass ratio according to the equipartition of energies. Recently, the corresponding state for a granular mixture was studied using a two component system of hard spheres with inelastic collisions [1]. Instead of the equilibrium state, the granular mixture attains a homogeneous cooling state (HCS) in which all time dependence occurs through a scaling of the particles velocities by their root mean square velocities. Although both components have a common cooling rate due to the inelastic collisions in the HCS, their granular temperatures are different. In terms of their mean square velocities, this implies a violation of the classical equipartition theorem. The extent of the violation depends on the mechanical differences of the particles (e.g., mass, diameter, coefficient of restitution), and is greatest when the differences are large. The quantitative predictions of the two temperatures from an Enskog-Lorentz kinetic theory have been confirmed by Monte Carlo simulations [2].

This effect also occurs for the simplest mixture of an impurity particle in a one component fluid. The impurity "equilibrates" to a common HCS with different temperatures for the impurity and fluid particles. The dynamics of an impurity particle of mass $m_{0}$ in a granular fluid with particles of mass $m$ has been studied for the limiting case of $m / m_{0} \ll 1[3]$. The description was based on the Enskog-Lorentz kinetic equation for the impurity in a dense fluid and the fluid was taken to be in its homogeneous cooling state (HCS). As for the case of elastic collisions, the kinetic equation reduces to a simple Fokker-Planck equation in this limit with a velocity independent friction coefficient. The solution to this equation approaches an HCS for the impurity particle. As expected, the kinetic temperatures of the two types of particles (defined in terms of their mean square velocities) are always different although their cooling rates are the same. The ratio of impurity to fluid thermal velocities is not simply $\mathrm{m} / \mathrm{m}_{0}$ as for equipartition of energy, but has a more complex mass dependence, according to the mechanical properties of both particles and the degree of inelasticity in collisions. Nevertheless, the analysis requires that this mass dependence be such that the ratio of thermal velocities should vanish for $m / m_{0} \rightarrow 0$ just as it would for equipartition. A single parameter $\xi^{*}$, the ratio of the cooling rate to the impurity-fluid particle collision rate, characterizes the domain for which the thermal velocity ratio vanishes, $\xi^{*}<1$. The predictions of the Fokker-Planck equation in this domain (velocity distribution, temperature ratio, mean square displacement, diffusion coefficient) have been confirmed by both Monte Carlo and molecular dynamics simulation [4]. As $\xi^{*} \rightarrow 1$ the diffusion coefficient calculated from this Fokker-Planck equation diverges.

The objective here is to put the analysis of reference [3] in context by extending the discussion to $\xi^{*} \geq 1$. A preliminary report of this work has been given in reference .5. It is found that there is a qualitative change in the state of the system at $\xi^{*}=1$ that is analogous to a second order phase transition. The order parameter $\bar{\phi}_{\mathrm{s}}$ is the 
ratio of thermal velocities with a conjugate field $h$ proportional to the mass ratio. The parameter $\xi^{*}$ is the analogue of the inverse temperature. The terminology "ordered" is used in analogy with magnetic systems where the ordered phase has a non-zero order parameter (magnetization) at zero external field. More precisely, the ordered phase here is associated with a broken symmetry or scaling $\lambda h \Rightarrow \lambda \bar{\phi}_{\mathrm{s}}$ which applies for $\xi^{*}<1$ but does not hold for $\xi^{*} \geq 1$. For $\xi^{*}<1$ the fluid is "normal" with $\bar{\phi}_{\mathrm{s}}=0$ at $h=0$, as in the case of a system with elastic collisions. For $\xi^{*}>1$ an "ordered" state with $\bar{\phi}_{\mathrm{s}} \neq 0$ occurs at $h=0$, representing an extreme breakdown of equipartition. Critical slowing and qualitative changes in the velocity distribution function for the impurity particle occur near the transition. The diffusion coefficient diverges for $\xi^{*} \geq 1$ and can be understood as a transition from diffusive to ballistic motion.

In the next section three characteristic frequencies are introduced: the cooling rate for the fluid particles, the cooling rate for the impurity, and the impurity-fluid collision rate. A simple estimate is obtained using a maximum entropy distribution to construct a phenomenological overview of the HCS, its properties for $m / m_{0} \ll 1$ (or equivalently $h \ll 1$ ), and the phase transition analogy. In Section IIII the diffusion coefficient is calculated from its Green-Kubo representation using the leading term in a cumulant expansion of the velocity autocorrelation function [6]. The diffusion coefficient is expressed as a function of the order parameter $\bar{\phi}_{\mathbf{S}}\left(\xi^{*}, h\right)$, and for $\xi^{*}<1$ the results of [3] are recovered. Otherwise, at the critical point and in the ordered phase, it is divergent. This divergence is interpreted by reconsideration of the Green-Kubo expression for finite times, showing a crossover from diffusive behavior in the normal phase to ballistic motion in the ordered phase.

A more complete description is given in Section IV based on an exact asymptotic analysis of the Enskog-Lorentz kinetic equation for the impurity particle velocity distribution function. This distribution function is calculated in the critical domain showing a crossover from Maxwellian for $\xi^{*}<1$ to an exponential quartic function of the velocity centered about a non-zero value for $\xi^{*}>1$. The functional form of $\bar{\phi}_{\mathbf{s}}\left(h, \xi^{*}\right)$ and associated critical properties are similar to those obtained in the phenomenological overview, with no qualitative differences. These results are summarized and discussed in the last section.

\section{PHENOMENOLOGICAL OVERVIEW}

Consider a fluid of hard, smooth, inelastic spheres of mass $m$, diameter $\sigma$, and fluid-fluid particle coefficient of normal restitution $\alpha$. In all of the following it is assumed that the fluid is in its HCS. Due to the inelastic collisions among particles the mean kinetic energy decreases as a function of time (referred to as "cooling"). An impurity particle of mass $m_{0}$, diameter $\sigma_{0}$, and impurity-fluid particle coefficient of restitution $\alpha_{0}$ is inserted in the fluid at some initial time. There is energy transfer between the impurity and fluid particles due to collisions and subsequently a common HCS for the fluid and impurity is attained where all particles have the same cooling rate. In this section a phenomenological but accurate description of this process and the HCS is given to present the basic ideas in a simple physical context.

\section{A. Nonlinear friction coefficient}

The primary property of interest is the ratio of the mean square velocities for the impurity and fluid particles

$$
\bar{\phi}(t)=\frac{\left\langle v_{0}^{2}(t)\right\rangle}{\left\langle v^{2}(t)\right\rangle},
$$

where the brackets denote an average over the initial state of the fluid plus impurity particle. This function measures the accommodation of the impurity particle to the fluid and will be referred to in the following as the order parameter. The cooling rates associated with the mean square velocities are defined by

$$
\xi(t)=-\partial_{t} \ln \left\langle v^{2}(t)\right\rangle, \quad \xi_{0}(t)=-\partial_{t} \ln \left\langle v_{0}^{2}(t)\right\rangle .
$$

For dimensionless units it is useful to define an average impurity-fluid particle collision rate

$$
\nu_{c}(t)=\frac{8}{3} h \rho \pi \bar{\sigma}^{2} g_{0}\langle v(t)\rangle, \quad h \equiv \frac{1+\alpha_{0}}{2} \frac{m}{m+m_{0}},
$$

where $\bar{\sigma}=\left(\sigma+\sigma_{0}\right) / 2$ is the average diameter, $\rho$ is the fluid density, $g_{0}$ is the pair correlation function for the impurity particle and a fluid particle at contact, and $\langle v(t)\rangle$ is the average speed of a fluid particle in the HCS. The parameter $h$ has been introduced as a measure of the mass ratio. As a function of $h$ this form for the collision frequency is the 
same as that for elastic collisions characterizing the equilibration rate. A dimensionless equation for $\bar{\phi}(t)$ now can be written in the form

$$
\partial_{s} \bar{\phi}=\left(\xi^{*}-\xi_{0}^{*}\right) \bar{\phi}
$$

where the dimensionless cooling rates and dimensionless time have been introduced as

$$
\xi^{*}=\frac{\xi}{\nu_{c}}, \quad \xi_{0}^{*}=\frac{\xi_{0}}{\nu_{c}}, \quad d s=\nu_{c}(t) d t
$$

To proceed it is necessary to calculate $\xi^{*}$ and $\xi_{0}^{*}$ as functions of $\bar{\phi}$. As shown in Appendix A, these are related to averages over the pair distribution function for two fluid particles and for a fluid and the impurity particle, respectively. This is a formal result since the distribution functions are not known. As a phenomenological estimate therefore, these averages are performed using a maximum entropy ensemble parameterized by the true mean square velocities. The qualitative accuracy of this approximation is confirmed in Section IV. The results of Appendix A are

$$
\xi^{*}=\frac{1-\alpha^{2}}{4 \sqrt{2} h} \frac{g}{g_{0}}\left(\frac{\sigma}{\bar{\sigma}}\right)^{2}, \quad \xi_{0}^{*}(\bar{\phi})=(1+\bar{\phi})^{1 / 2}\left(1-h \frac{1+\bar{\phi}}{\bar{\phi}}\right)
$$

where $g$ is the pair correlation function for two fluid particles at contact. This form for the cooling rate $\xi_{0}^{*}$ of the impurity is the same as that for elastic collisions and represents the equilibration rate. The new features of inelasticity are primarily described by $\xi^{*}$, which is independent of $\bar{\phi}$. The equation for $\bar{\phi}(s)$ with these approximate forms for the cooling rates is

$$
\left[\partial_{s}+\bar{\gamma}^{*}(\bar{\phi})-\xi^{*}\right] \bar{\phi}=h \bar{n}(\bar{\phi}),
$$

which results from the decomposition $\xi_{0}^{*}(\bar{\phi})=\bar{\gamma}^{*}(\bar{\phi})-h \bar{n}^{*}(\bar{\phi}) / \bar{\phi}$ with the definitions

$$
\bar{\gamma}^{*}(\bar{\phi})=(1+\bar{\phi})^{1 / 2}, \quad \bar{n}(\bar{\phi})=(1+\bar{\phi})^{3 / 2} .
$$

This has the same form as would be obtained from a simple Langevin or Brownian motion model where $\bar{\gamma}^{*}(\bar{\phi})$ is the "friction constant" or nonlinear impurity-fluid collision frequency and $h \bar{n}(\bar{\phi})$ is the noise amplitude. The solution to this equation is a function of time and the two parameters $\xi^{*}$ and $h$. The stationary solutions $\bar{\phi}_{\mathbf{s}}\left(\xi^{*}, h\right)$ are determined from

$$
\bar{\phi}_{\mathrm{s}}=h \frac{\bar{n}\left(\bar{\phi}_{\mathrm{s}}\right)}{\bar{\gamma}^{*}\left(\bar{\phi}_{\mathrm{s}}\right)-\xi^{*}} .
$$

This form shows most clearly the effect of competition between "friction" on the impurity particle and fluid cooling since $\bar{\gamma}^{*}\left(\bar{\phi}_{\mathrm{s}}\right)>\xi^{*}$ is required for positive, finite solutions. This generalizes the result obtained in reference [3] which is limited to $\xi^{*}<1$ and $h \rightarrow 0$. It is easily verified that a unique positive solution to (9) exists for all positive $\xi^{*}$ and $h$ and that it is linearly stable. The latter confirms that the HCS characterized by $\bar{\phi}_{\mathrm{s}}$ is approached for long times for a wide class of initial conditions. The time scale for formation of the HCS is discussed below. For elastic collisions $\left(\alpha=\alpha_{0}=1\right)$ the solution is $\bar{\phi}_{\mathrm{s}}=h /(1-h)=m / m_{0}$ as required by equipartition. If only the impurity-fluid particle collisions are inelastic (i.e., $\left.\alpha=1, \xi^{*}=0\right)$ a recent result of Martin and Piasecki is recovered [7], $\bar{\phi}_{\mathrm{s}}=h /(1-h)=m\left(1+\alpha_{0}\right) /\left[2 m_{0}+m\left(1-\alpha_{0}\right)\right]$.

More generally, Eq. (9) can be transformed into a cubic equation for $\bar{\phi}_{\mathrm{s}}$ whose physical solution gives $\bar{\phi}_{\mathrm{s}}\left(\xi^{*}, h\right)$ for arbitrary $\xi^{*}$ and $h$. Figure 1 shows $\bar{\phi}_{\mathbf{s}}\left(\xi^{*}, h\right)$ as a function of $h$ for $\xi^{*}=0.9,1.0$, and 1.1. An instructive alternative form for the determination of $\bar{\phi}_{\mathrm{s}}$ is

$$
\xi^{*}=\xi_{0}^{*}\left(\bar{\phi}_{\mathrm{s}}\right)
$$

The graphical solution to Eq. (10) is obtained in a plane $y$ vs $\bar{\phi}$ by finding the value of $\bar{\phi}$ at which the constant $y=\xi^{*}$ intercepts the curve $y=\xi_{0}^{*}(\bar{\phi})$, as illustrated in Fig. 2. There is seen to be a qualitative difference between the solutions for $\xi^{*}<1$ and $\xi^{*}>1$ in the limit of small $h$. Since $\bar{\gamma}^{*}(\bar{\phi}) \geq \bar{\gamma}^{*}(0)=1$ and $\bar{n}(0)=1$ the asymptotic solution for $\xi^{*}<1$ is

$$
\bar{\phi}_{\mathrm{s}} \rightarrow h \frac{\bar{n}(0)}{\bar{\gamma}^{*}(0)-\xi^{*}}=\frac{h}{1-\xi^{*}},
$$


which agrees with [3]. The mechanism responsible for solutions with $\xi^{*}>1$ is now clear. As $\xi^{*}$ exceeds $\bar{\gamma}^{*}(0)$ the nonlinear dependence of the friction coefficient on $\bar{\phi}$ is activated to maintain positivity of $\bar{\gamma}^{*}\left(\bar{\phi}_{\mathrm{S}}\right)-\xi^{*}$. Since $\bar{\gamma}^{*}(\bar{\phi})$ is a monotonically increasing function of $\bar{\phi}$, positivity is possible for any choice of $\xi^{*}$. In general this requires that $\bar{\phi}$ must be finite even for $h \rightarrow 0$. This is possible if $\bar{\gamma}^{*}\left(\bar{\phi}_{\mathrm{s}}\right)-\xi^{*}$ is of order $h$ for small $h$ or $\bar{\phi}_{\mathrm{s}}=$ constant $+\mathcal{O}(h)$. This nonlinear dependence of the friction coefficient on $\bar{\phi}_{\mathrm{s}}$ provides the mechanism whereby the coupling of the impurity particle to the fluid can be enhanced for large cooling rates: the impurity-fluid collision frequency is increased by an increased mean square velocity of the impurity relative to that of the fluid. This is illustrated in Figs. 1 and 2 showing the qualitative difference between $\xi^{*}<1$ and $\xi^{*}>1$. The former admits $\bar{\phi}_{\mathrm{s}} \rightarrow 0$ for $h \rightarrow 0$ whereas the latter requires $\bar{\phi}_{\mathrm{s}}=$ constant. In more detail, the asymptotic solution to (9) is

$$
\bar{\phi}_{\mathrm{s}}\left(\xi^{*}, h\right) \rightarrow \begin{cases}\left(1-\xi^{*}\right)^{-1} h, & \xi^{*}<1 \\ \sqrt{2 h}, & \xi^{*}=1 \\ \xi^{* 2}-1+2 \xi^{* 4}\left(\xi^{* 2}-1\right)^{-1} h, & \xi^{*}>1\end{cases}
$$

The common domain of $h \rightarrow 0$ and $\xi^{*} \rightarrow 1$ can be obtained from (9) by the scaling $h \rightarrow \epsilon^{2} h, \bar{\phi}_{\mathrm{s}} \rightarrow \epsilon \bar{\phi}_{\mathrm{s}}$, and $1-\xi^{*} \rightarrow \epsilon\left(1-\xi^{*}\right)$, for $\epsilon \ll 1$. The result is the quadratic form

$$
h \approx\left(1-\xi^{*}\right) \bar{\phi}_{\mathrm{s}}+\frac{1}{2} \bar{\phi}_{\mathrm{s}}^{2}
$$

which has the solution

$$
\bar{\phi}_{\mathrm{s}} \approx \xi^{*}-1+\sqrt{\left(\xi^{*}-1\right)^{2}+2 h}
$$

At $h=0$ this gives $\bar{\phi}_{\mathrm{s}} \approx \xi^{*}-1+\left|\xi^{*}-1\right|$, illustrating again the qualitative difference between $\xi^{*}<1$ and $\xi^{*}>1$.

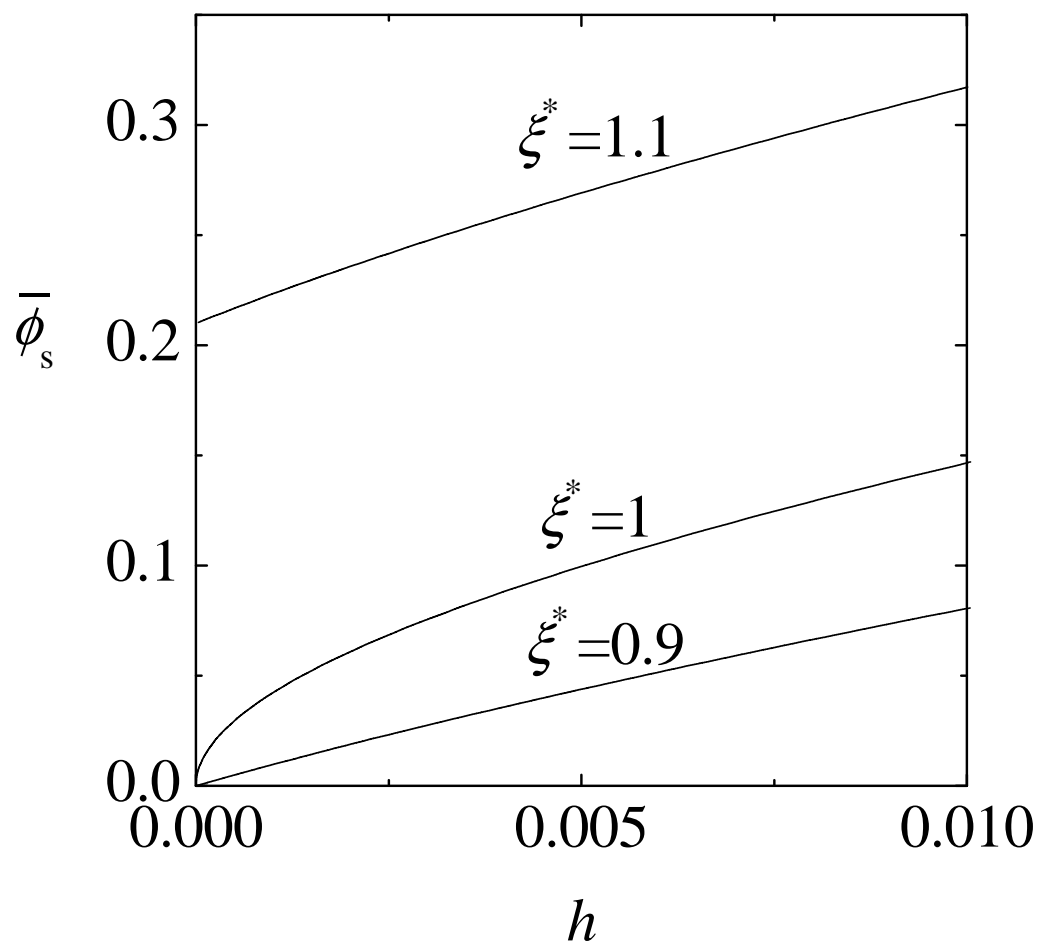

FIG. 1. Ratio of mean square velocities, $\bar{\phi}_{\mathrm{s}}$, as a function of the mass ratio parameter $h$ for $\xi^{*}=0.9,1$, and 1.1 . 


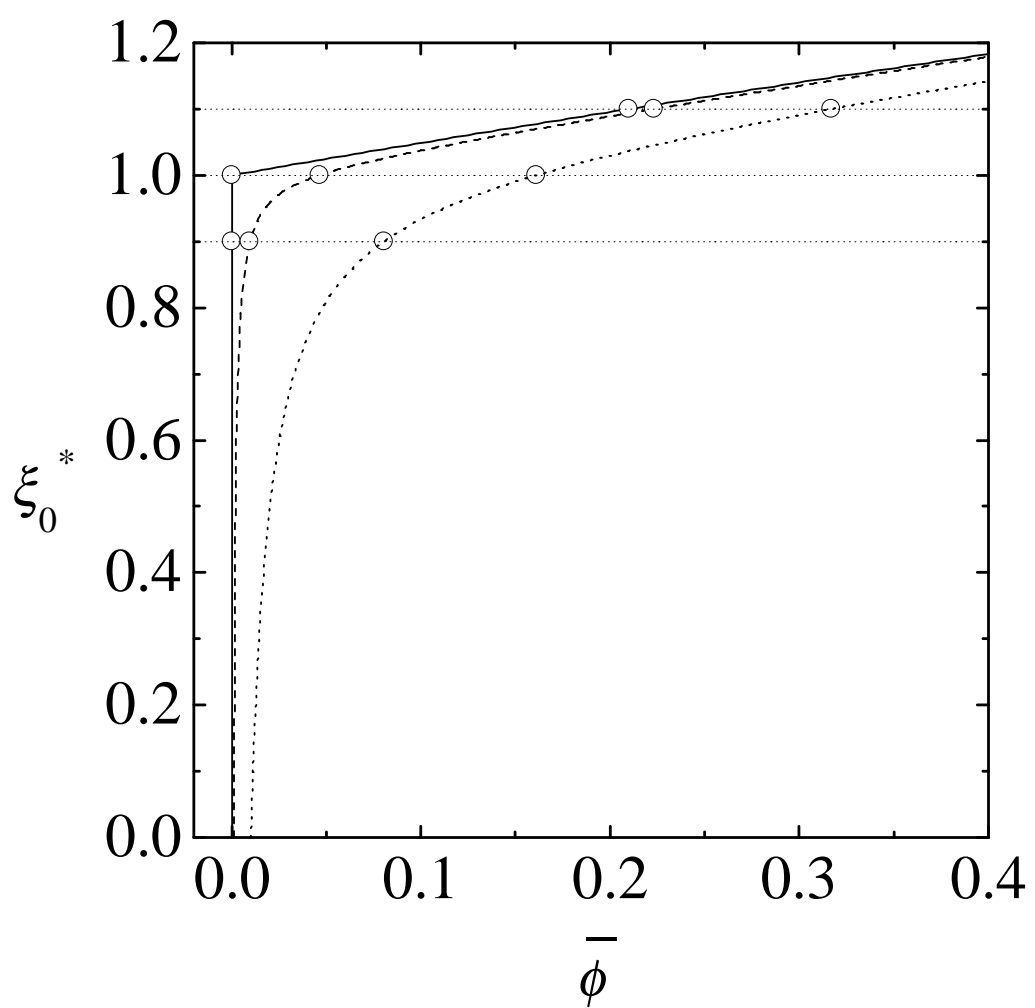

FIG. 2. Plot of $\xi_{0}^{*}(\bar{\phi})$, Eq. ( $(\overline{6})$, for $h=10^{-2}$ (dotted line), $h=10^{-3}$ (dashed line), and $h=0$ (solid line). The intercepts of the curves with the horizontal lines $\xi^{*}=0.9,1$, and 1.1 give the corresponding values of $\bar{\phi}_{\mathrm{s}}\left(\xi^{*}, h\right)($ circles $)$.

Figures 1 and 2 show that the fluid cooling rate relative to the impurity-fluid collision rate $\xi^{*}$ is a control parameter distinguishing different dependencies of $\bar{\phi}_{\mathrm{s}}$ on $h$ for small $h$. This will be exploited in the next subsection, where $\xi^{*}=1$ identifies a critical point. Since $\xi^{*} \propto\left(1-\alpha^{2}\right) / h$, Eq. (16), the plots of $\bar{\phi}_{\mathrm{s}}$ at constant $\xi^{*}$ require the change of both the fluid coefficient of restitution $\alpha$ and the mass ratio parameter $h$. It is instructive, however, to examine the mean square velocity ratio $\bar{\phi}_{\mathrm{s}}$ and the mean energy ratio $\epsilon_{0} / \epsilon=\left(m_{0} / m\right) \bar{\phi}_{\mathrm{s}}$ as functions of $h$ at fixed $\alpha<1$. In that case, $\xi^{*} \sim h^{-1}$ diverges in the limit $h \rightarrow 0$ and so do $\bar{\phi}_{\mathrm{s}} \approx \xi^{* 2}(1+h)^{2}-1 \sim h^{-2}$ and $\epsilon_{0} / \epsilon \sim h^{-3}$. This is illustrated in Fig. 3, where $\bar{\phi}_{\mathrm{s}}$ and $\epsilon_{0} / \epsilon$ are plotted versus $h$ for $\alpha=1,0.99$, and 0.95 (taking, for simplicity, $\alpha_{0}=1, \sigma=\sigma_{0}, g=g_{0}=1$ ). The dotted lines for $\alpha=1$ represent equipartition for which $\bar{\phi}_{\mathrm{s}} \rightarrow h /(1-h)$ and $\epsilon_{0} / \epsilon \rightarrow 1$. For $\alpha<1$ there is a sharp deviation at sufficiently small $h$ representing the crossover to the domain for which $\xi^{*}>1$. 


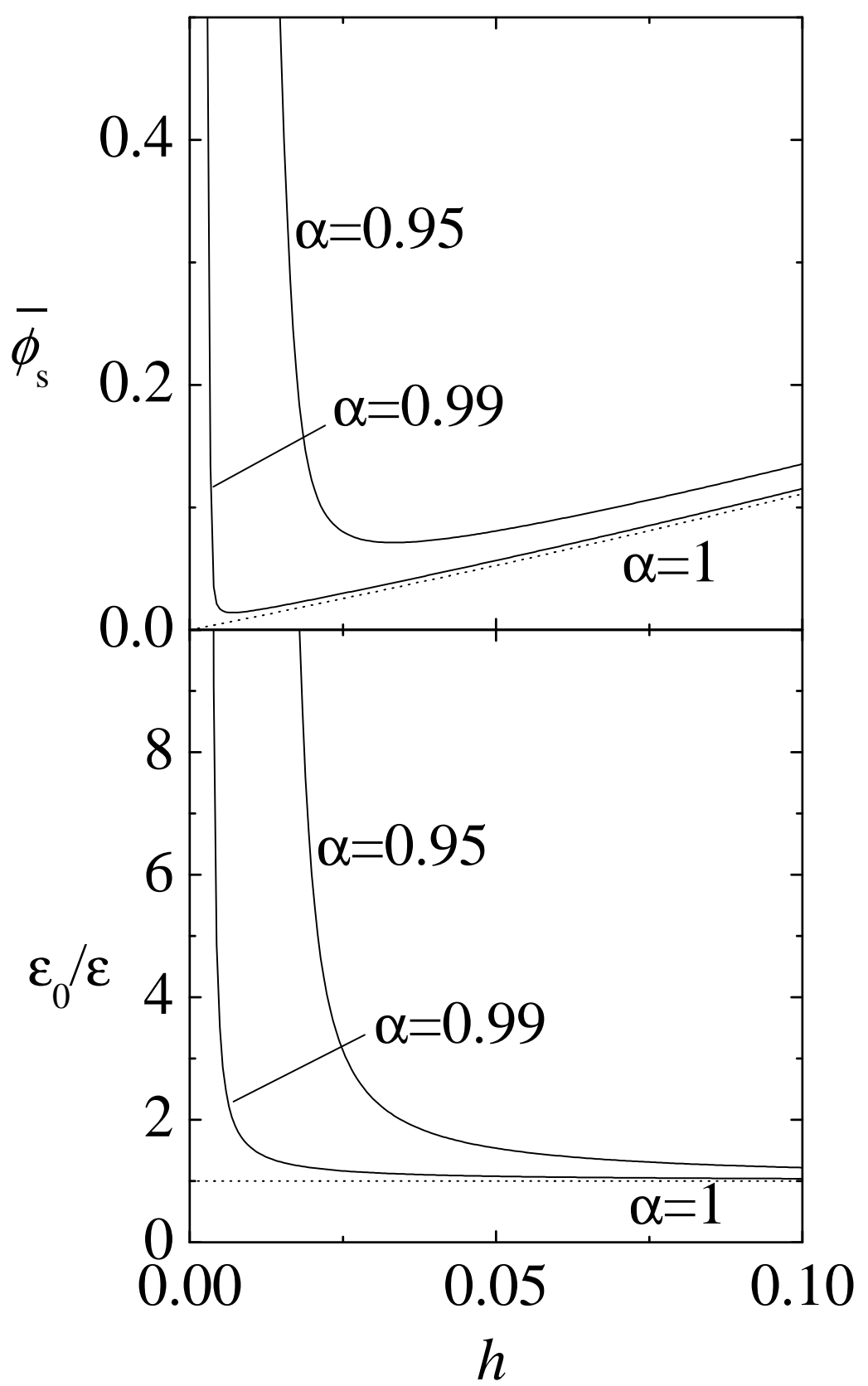

FIG. 3. Ratio of mean square velocities, $\bar{\phi}_{\mathrm{s}}$, and of mean kinetic energies, $\epsilon_{0} / \epsilon$, as functions of the mass ratio parameter $h$ for $\alpha=1,0.95$, and 0.99 .

\section{B. Representation as a phase transition}

Figure 1 and Eqs. (12) and (14) are reminiscent of the thermodynamics for magnetization as a function of an external magnetic field. Below some critical temperature the magnetization is finite at zero field, while above that temperature it vanishes at zero field. To pursue this analogy, consider $\bar{\phi}_{\mathbf{s}}\left(\xi^{*}, h\right)$ as the order parameter (magnetization), $h$ the conjugate field (magnetic field), and $\xi^{*}$ as the control parameter (inverse temperature). The "equation of state" for the system is obtained from (9) by solving for $h\left(\bar{\phi}_{\mathrm{s}}, \xi^{*}\right)$,

$$
h\left(\bar{\phi}_{\mathrm{s}}, \xi^{*}\right)=\frac{\bar{\phi}_{\mathrm{s}}}{\left(1+\bar{\phi}_{\mathrm{s}}\right)^{3 / 2}}\left[\left(1+\bar{\phi}_{\mathrm{s}}\right)^{1 / 2}-\xi^{*}\right] .
$$


A Helmholtz free energy can be defined in the usual way

$$
F\left(\bar{\phi}_{\mathrm{s}}, \xi^{*}\right)=\int_{0}^{\bar{\phi}_{\mathrm{s}}} d x h\left(x, \xi^{*}\right)=\bar{\phi}_{\mathrm{s}}-\ln \left(1+\bar{\phi}_{\mathrm{s}}\right)-2 \xi^{*}\left[\frac{2+\bar{\phi}_{\mathrm{s}}}{\left(1+\bar{\phi}_{\mathrm{s}}\right)^{1 / 2}}-2\right]
$$

Next, the Gibbs free energy is obtained from the Legendre transformation

$$
\begin{aligned}
\Phi\left(\xi^{*}, h\right) & =F\left(\bar{\phi}_{\mathrm{s}}, \xi^{*}\right)-h \bar{\phi}_{\mathbf{s}}\left(\xi^{*}, h\right) \\
& =(1-h) \bar{\phi}_{\mathbf{s}}\left(\xi^{*}, h\right)-\ln \left[1+\bar{\phi}_{\mathbf{s}}\left(\xi^{*}, h\right)\right]-2 \xi^{*}\left\{\frac{2+\bar{\phi}_{\mathbf{s}}\left(\xi^{*}, h\right)}{\left[1+\bar{\phi}_{\mathbf{s}}\left(\xi^{*}, h\right)\right]^{1 / 2}}-2\right\} .
\end{aligned}
$$

The first and second derivatives of $\Phi\left(\xi^{*}, h\right)$ provide the order parameter $\bar{\phi}_{\mathrm{s}}$, "entropy" $\Sigma$, "susceptibility" $\chi$, "expansion coefficient" $\alpha_{h}$, and "heat capacity" $C_{h}$. The results are

$$
\begin{gathered}
\Sigma\left(\xi^{*}, h\right)=\frac{\partial \Phi\left(\xi^{*}, h\right)}{\partial \xi^{*}}=-2\left[\frac{2+\bar{\phi}_{\mathrm{s}}}{\left(1+\bar{\phi}_{\mathrm{s}}\right)^{1 / 2}}-2\right] \\
\chi\left(\xi^{*}, h\right)=-\frac{\partial^{2} \Phi\left(\xi^{*}, h\right)}{\partial h^{2}}=\frac{\partial \bar{\phi}_{\mathrm{s}}\left(\xi^{*}, h\right)}{\partial h}=\frac{\left(1+\bar{\phi}_{\mathrm{s}}\right)^{5 / 2}}{\left(1+\bar{\phi}_{\mathrm{s}}\right)^{1 / 2}-\xi^{*}\left(1-\frac{1}{2} \bar{\phi}_{\mathrm{s}}\right)}, \\
\alpha_{h}\left(\xi^{*}, h\right)=-\frac{\partial^{2} \Phi\left(\xi^{*}, h\right)}{\partial \xi^{*} \partial h}=\frac{\partial \bar{\phi}_{\mathrm{s}}\left(\xi^{*}, h\right)}{\partial \xi^{*}}=\chi \frac{\bar{\phi}_{\mathrm{s}}}{\left(1+\bar{\phi}_{\mathrm{s}}\right)^{3 / 2}}, \\
C_{h}\left(\xi^{*}, h\right)=-\frac{\partial^{2} \Phi\left(\xi^{*}, h\right)}{\partial \xi^{* 2}}=\chi^{-1} \alpha_{h}^{2} .
\end{gathered}
$$

The values of these thermodynamic properties in the limit $h=0$ for $\xi^{*} \neq 1$ follow directly from the asymptotic forms (12) for $\bar{\phi}_{\mathrm{s}}$ :

$$
\begin{aligned}
& \Phi\left(\xi^{*}, h=0\right)= \begin{cases}0, & \xi^{*}<1, \\
\left(\xi^{*}-1\right)\left(3-\xi^{*}\right)-2 \ln \xi^{*}, & \xi^{*}>1 .\end{cases} \\
& \Sigma\left(\xi^{*}, h=0\right)= \begin{cases}0, & \xi^{*}<1, \\
-2 \frac{\left(\xi^{*}-1\right)^{2}}{\xi^{*}}, & \xi^{*}>1,\end{cases} \\
& \chi\left(\xi^{*}, h=0\right)=\frac{1}{\left|\xi^{*}-1\right|} \begin{cases}1, & \xi^{*}<1, \\
\frac{2 \xi^{* 4}}{\xi^{*}+1}, & \xi^{*}>1,\end{cases} \\
& \alpha_{h}\left(\xi^{*}, h=0\right)= \begin{cases}0, & \xi^{*}<1 \\
2 \xi^{*}, & \xi^{*}>1\end{cases} \\
& C_{h}\left(\xi^{*}, h=0\right)= \begin{cases}0, & \xi^{*}<1, \\
2 \frac{\xi^{* 2}-1}{\xi^{* 2}}, & \xi^{*}>1 .\end{cases}
\end{aligned}
$$

With the exception of $\chi$, all thermodynamic variables vanish for $\xi^{*}<1$ and are finite for $\xi^{*}>1$. All are continuous at $\xi^{*}=1$, except for $\alpha_{h}$ which has a finite discontinuity. The susceptibility diverges as $\left|\xi^{*}-1\right| \rightarrow 0$. Thus either the discontinuity of $\alpha_{h}$ or the divergence of $\chi$ characterizes a second order phase transition at $\xi^{*}=1$. Since the order parameter $\bar{\phi}_{\mathrm{s}}$ behaves qualitatively as that for a system with elastic collisions when $\xi^{*}<1$, this will be referred to as 
the "normal" phase. In contrast, since $\bar{\phi}_{\mathrm{s}} \neq 0$ for $\xi^{*}>1$ this will be called the "ordered" phase. The entropy function $\Sigma\left(\xi^{*}, h\right)$ is plotted versus $\xi^{*}$ for $h=10^{-2}, 10^{-3}$, and 0 in Fig. 4 . The negative value of $\Sigma$ at $h=0$ and $\xi^{*}>1$ is a measure of the degree of "order" in the ordered phase. The response functions $\chi, \alpha_{h}$, and $C_{h}$ at $h=0,10^{-2}$, and

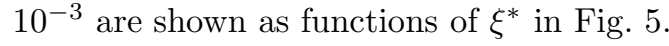

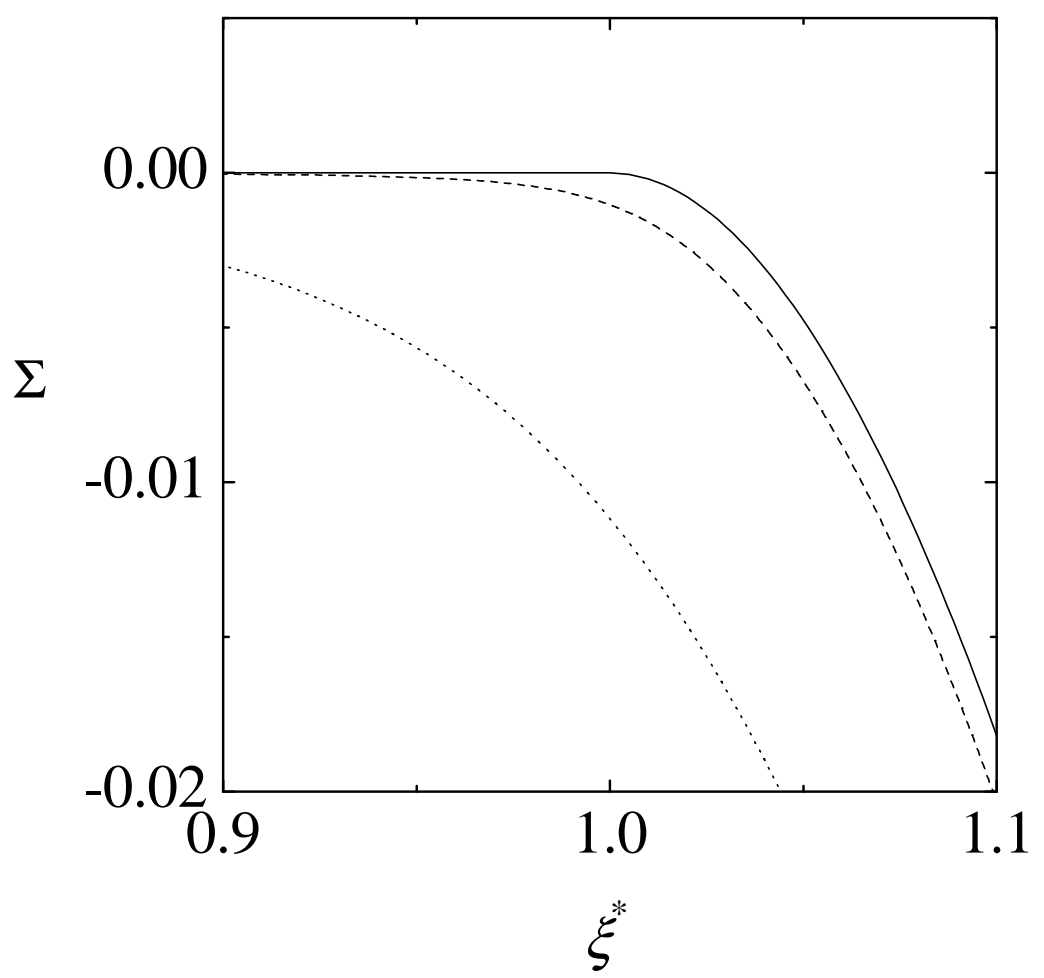

FIG. 4. Entropy as a function of $\xi^{*}$ for $h=10^{-2}$ (dotted line), $h=10^{-3}$ (dashed line), and $h=0$ (solid line). 


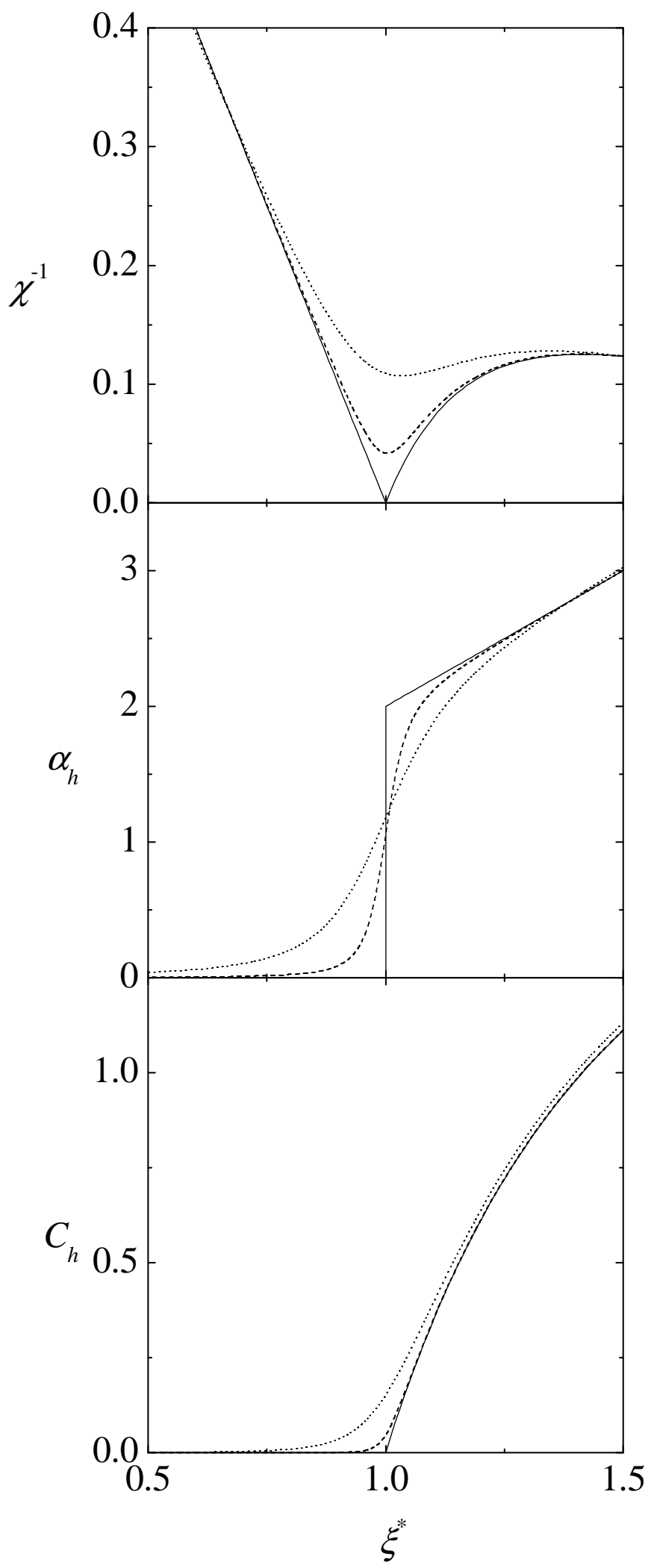

FIG. 5. Inverse susceptibility $\left(\chi^{-1}\right)$, expansion coefficient $\left(\alpha_{h}\right)$, and heat capacity $\left(C_{h}\right)$ as functions of $\xi^{*}$ for $h=10^{-2}$ (dotted line), $h=10^{-3}$ (dashed line), and $h=0$ (solid line). 
Near the critical region $\left(h \ll 1,\left|\xi^{*}-1\right| \ll 1\right)$, the free energy adopts the Landau-like form

$$
\Phi\left(\xi^{*}, h\right) \approx \frac{1}{2}\left(1-\xi^{*}\right) \bar{\phi}_{\mathrm{s}}^{2}+\frac{1}{6} \bar{\phi}_{\mathrm{s}}^{3}-h \bar{\phi}_{\mathrm{s}}
$$

which yields the critical equation of state (13), as expected. It is easily verified that the free energy and the equation of state in the critical region satisfy the scaling relations

$$
\Phi\left(\lambda\left(\xi^{*}-1\right), \lambda^{a} h\right)=\lambda^{b} \Phi\left(\xi^{*}-1, h\right), \quad \bar{\phi}_{\mathbf{s}}\left(\lambda\left(\xi^{*}-1\right), \lambda^{a} h\right)=\lambda^{b-a} \bar{\phi}_{\mathbf{s}}\left(\xi^{*}-1, h\right)
$$

with $a=2$ and $b=3$. These scaling relations suffice to determine the critical exponents [8] $\hat{\delta}=a /(b-a)=2$, $\hat{\beta}=b-a=1$, and $\hat{\gamma}=2 a-b=1$, while the critical exponent $\hat{\alpha}=2-b=-1$ is negative, indicating that $C_{h}$ is continuous at the critical point.

\section{Critical dynamics}

If the ratio between the initial mean square velocities of the fluid and impurity particles is not that given by the solution to (9), there is an evolution to the HCS described by (7) which can be written in the Ginzburg-Landau form

$$
\partial_{s} \bar{\phi}=-\bar{n}(\bar{\phi}) \frac{\partial \Phi\left(\xi^{*}, h ; \bar{\phi}\right)}{\partial \bar{\phi}} .
$$

Here, $\Phi\left(\xi^{*}, h ; \bar{\phi}\right)$ is a variational free energy given by Eq. (17) with the order parameter $\bar{\phi}$ considered as an independent variable, and the kinetic coefficient is $\bar{n}(\bar{\phi})$. The stationary solution occurs for $\partial \Phi\left(\xi^{*}, h ; \bar{\phi}\right) / \partial \bar{\phi}=0$, which is just Eq. (9). It follows directly from (17) and (29) that $\Phi\left(\xi^{*}, h ; \bar{\phi}\right)$ has the properties

$$
\Phi\left(\xi^{*}, h ; \bar{\phi}\right) \geq \Phi\left(\xi^{*}, h ; \bar{\phi}_{\mathrm{s}}\right), \quad \partial_{s} \Phi\left(\xi^{*}, h ; \bar{\phi}\right)=-\bar{n}(\bar{\phi})\left[\frac{\partial \Phi\left(\xi^{*}, h ; \bar{\phi}\right)}{\partial \bar{\phi}}\right]^{2} \leq 0 .
$$

This shows that $\Phi\left(\xi^{*}, h ; \bar{\phi}\right)$ is a Lyapunov function for the dynamics: it is bounded from below by the HCS solution and monotonically approaches this bound. Consequently, the HCS solution results in both phases for a wide class of homogeneous initial conditions and is stable.

The free energy $\Phi\left(\xi^{*}, h=0 ; \bar{\phi}\right)$ is shown in Fig. 6 for $\xi^{*}=0.9,1$, and 1.1. As expected, the minimum is located at $\bar{\phi}=0$ for $\xi^{*} \leq 1$ and at $\bar{\phi} \neq 0$ for $\xi^{*}>1$. For states near the HCS the evolution equation (29) can be linearized and a characteristic response time $\tau^{*}$ identified according to

$$
-\partial_{s} \ln \left|\bar{\phi}-\bar{\phi}_{\mathrm{s}}\right|=\tau^{*-1}=\left(\bar{n} \chi^{-1}\right)_{\bar{\phi}_{\mathrm{s}}}
$$

In the elastic limit $\tau^{*}$ is just the equilibration time (in terms of the number of impurity-fluid particle collisions) for the impurity particle to attain a mean kinetic energy equal to that of the fluid particles. Similarly, for inelastic collisions it is the time for the impurity particle to reach a cooling rate equal to that of the fluid. This characteristic time is a smooth function of $h$ and $\xi^{*}$ except in the limit $h \rightarrow 0$ where $\tau^{*}$ diverges at $\xi^{*}=1$. This critical slowing follows directly from the fact that $\tau^{*} \propto \chi$. Otherwise, the relaxation times away from $\xi^{*}=1$ are finite and comparable for the normal and ordered states. Figure 7 shows the dependence of $\tau^{*-1}$ on $h$ for $\xi^{*}=0.9,1$, and 1.1. 


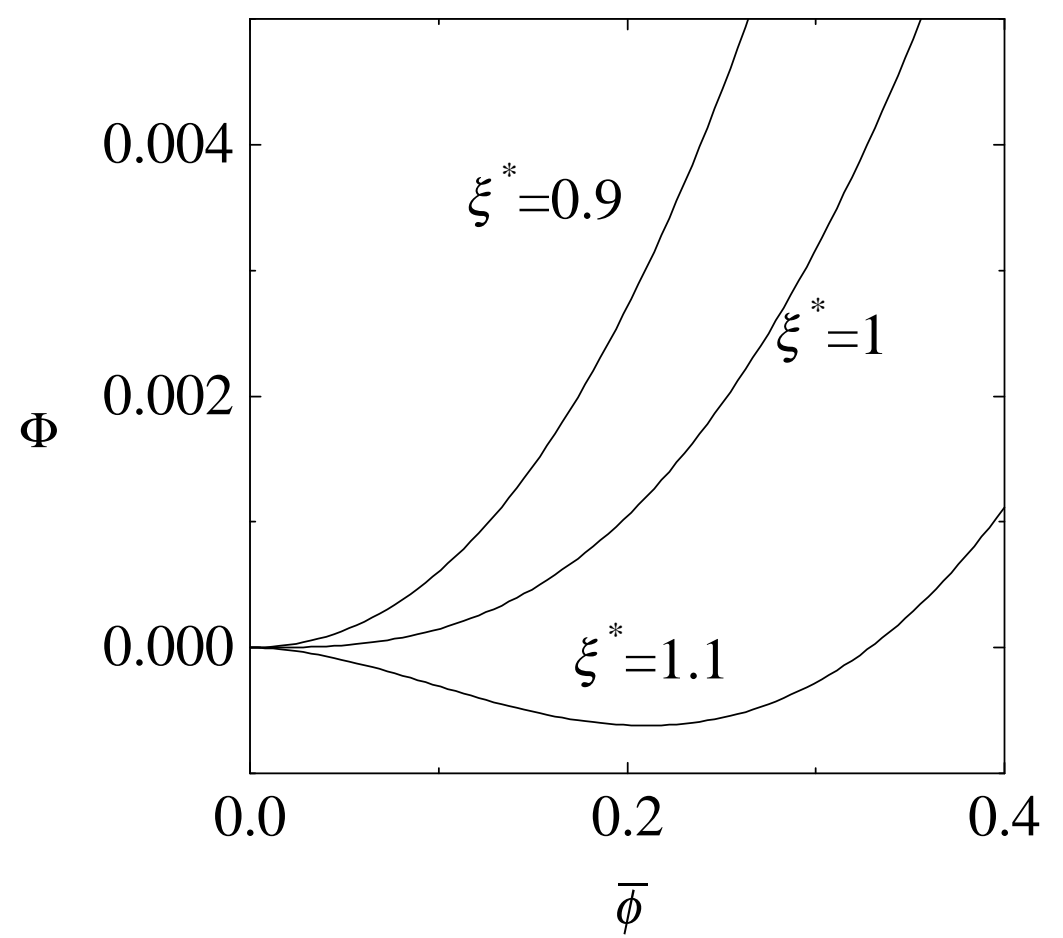

FIG. 6. Variational free energy $\Phi\left(\xi^{*}, h=0 ; \bar{\phi}\right)$ for $\xi^{*}=0.9,1$, and 1.1 .

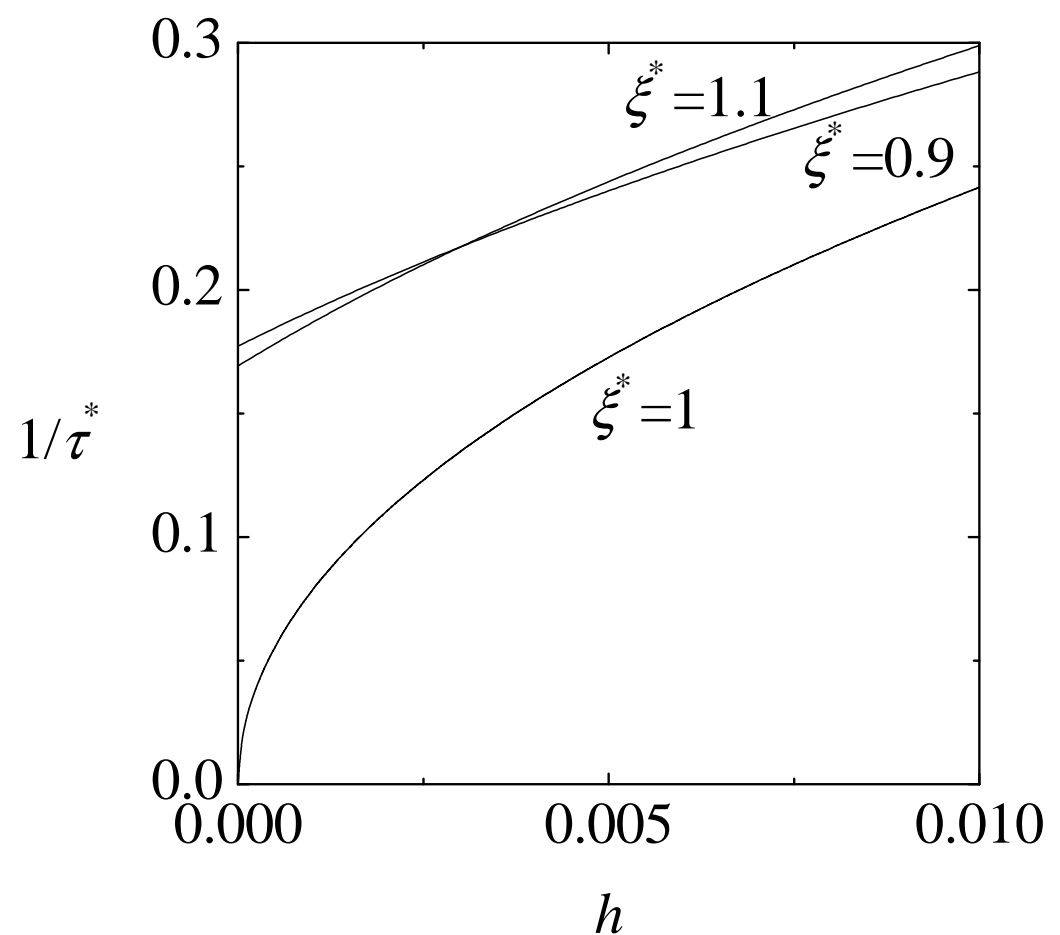

FIG. 7. Inverse characteristic time $\tau^{*-1}$ as a function of the mass ratio parameter $h$ for $\xi^{*}=0.9,1$, and 1.1 . 


\section{DIFFUSION}

Diffusion of an impurity particle in the HCS has been described in general elsewhere [6,9, 10, In this section the consequences for $h \rightarrow 0$ in the two phases are explored. A generalized diffusion equation can be obtained by extending the familiar methods of linear response to the granular fluid, which for long wavelengths takes the form

$$
\partial_{s} n^{*}\left(\mathbf{r}^{*}, s\right)-D^{*}(s) \nabla^{2} n^{*}\left(\mathbf{r}^{*}, s\right)=0 .
$$

Here $n^{*}\left(\mathbf{r}^{*}, s\right)$ is the dimensionless probability density to find the impurity particle at position $\mathbf{r}=\mathbf{r}^{*} \ell$, where $\ell=\left\langle v_{0}^{2}\right\rangle_{\mathrm{s}}^{1 / 2} / \nu_{c}$ is an effective mean free path, and $s$ is the dimensionless time of (5). The time dependent diffusion function $D^{*}(s)$ is given exactly by a Green-Kubo expression

$$
D^{*}(s)=\frac{1}{3\left\langle v_{0}^{* 2}\right\rangle_{\mathrm{s}}} \int_{0}^{s} d s^{\prime}\left\langle\mathbf{v}_{0}^{*}\left(s^{\prime}\right) \cdot \mathbf{v}_{0}^{*}\right\rangle_{\mathrm{s}},
$$

where $\mathbf{v}_{0}^{*}=\mathbf{v}_{0} / \sqrt{\left\langle v^{2}\right\rangle_{\mathrm{s}}}$ and the brackets denote an average over the dimensionless HCS ensemble. A phenomenological but accurate evaluation of the velocity autocorrelation function is given by its exact short time behavior

$$
\begin{aligned}
& \left\langle\mathbf{v}_{0}^{*}\left(s^{\prime}\right) \cdot \mathbf{v}_{0}^{*}\right\rangle_{\mathrm{s}} \rightarrow\left\langle v_{0}^{* 2}\right\rangle_{\mathrm{s}} e^{-\omega_{D}^{*} s^{\prime}}, \\
& \omega_{D}^{*}=-\frac{1}{2} \xi_{0}^{*}-\frac{\left\langle\left(L^{*} \mathbf{v}_{0}^{*}\right) \cdot \mathbf{v}_{0}^{*}\right\rangle_{\mathrm{s}}}{\left\langle v_{0}^{* 2}\right\rangle_{\mathrm{s}}},
\end{aligned}
$$

where $L^{*}$ is the dimensionless Liouville operator [cf. Appendix A]. The dimensionless frequency $\omega_{D}^{*}$ is calculated in Appendix A using the same approximation as that for the cooling rates in Section III, with the result

$$
\omega_{D}^{*}=\frac{1}{2}\left[\bar{\gamma}^{*}\left(\bar{\phi}_{\mathrm{s}}\right)-\xi_{0}^{*}\right]
$$

For a fluid with elastic collisions the approximation (34) coincides with that obtained from the Enskog-Lorentz equation in the first Sonine approximation, and is known to be accurate even for moderately dense systems. It is assumed that a similar level of accuracy extends to the inelastic case as well [10]. The diffusion function $D^{*}(s)$ becomes

$$
D^{*}(s)=\frac{1}{3 \omega_{D}^{*}}\left(1-e^{-\omega_{D}^{*} s}\right) .
$$

The analysis of Appendix $\mathrm{A}$ shows that $\omega_{D}^{*}>0$ for all finite $h$. Thus for $s \gg \omega_{D}^{*-1}$

$$
D^{*}(s) \rightarrow D^{*}=\frac{1}{3 \omega_{D}^{*}}
$$

and (32) becomes the usual diffusion equation with diffusion constant $D^{*}$. The initial transient period is the expected "ageing" required for applicability of hydrodynamics (diffusion).

Consider now the behavior as $h \rightarrow 0$. Using (8) and (9) $\omega_{D}^{*}$ can be expressed entirely in terms of $\bar{\phi}_{\mathrm{s}}$ and $h$

$$
\omega_{D}^{*}=\frac{1}{2} h \frac{\left(1+\bar{\phi}_{\mathrm{s}}\right)^{3 / 2}}{\bar{\phi}_{\mathrm{s}}} .
$$

Using (12) this frequency behaves for $h \rightarrow 0$ as

$$
\omega_{D}^{*}\left(\xi^{*}, h\right) \rightarrow \begin{cases}\frac{1}{2}\left(1-\xi^{*}\right), & \xi^{*}<1 \\ \frac{1}{2} \sqrt{\frac{h}{2}}, & \xi^{*}=1 \\ \frac{1}{2} \frac{\xi^{* 3}}{\xi^{* 2}-1} h, & \xi^{*}>1\end{cases}
$$

In general, $\omega_{D}^{*}\left(\xi^{*}, 0\right)$ is finite below the critical point, but vanishes at and above the critical point for $h=0$. Thus diffusion in the sense of (38) occurs at $h=0$ only for $\xi^{*}<1$. To understand the phenomenon for $\xi^{*} \geq 1$ note that for $\omega_{D}^{*}=0$ Eq. (37) becomes 


$$
D^{*}(s)=\frac{s}{3} .
$$

To interpret this, take the second moment of (32) with respect to $r^{2}$ to relate $D^{*}(s)$ to the mean square displacement of the impurity particle,

$$
D^{*}(s)=\frac{1}{6} \partial_{s}\left\langle\left|\mathbf{r}^{*}(s)-\mathbf{r}^{*}(0)\right|^{2}\right\rangle_{\mathrm{s}} .
$$

Thus the mean square displacement behaves as

$$
\left\langle\left|\mathbf{r}^{*}(s)-\mathbf{r}^{*}(0)\right|^{2}\right\rangle_{\mathrm{s}} \rightarrow \begin{cases}6 D^{*} s, & \xi^{*}<1 \\ s^{2}, & \xi^{*} \geq 1\end{cases}
$$

This shows that the impurity is not diffusing but rather undergoing ballistic motion at its root mean square speed if $\xi^{*} \geq 1$.

\section{ASYMPTOTIC KINETIC THEORY}

The analysis of Sections II and III is based on the plausible but uncontrolled estimate of the cooling rates for the fluid and impurity particles using a maximum entropy ensemble (Appendix A). Furthermore, it is limited to a discussion of the order parameter and diffusion but does not address other properties such as the velocity distribution itself. In this section the results of Section II are recovered systematically and with additional detail from the Enskog-Lorentz kinetic equation for the impurity particle velocity distribution [1].36] []. The features of interest here occur for $h \rightarrow 0$ so only an asymptotic representation of the kinetic theory is required. The fluid particle distribution is independent of $h$ and its detailed form is not required for the analysis here. The asymptotic form of the Enskog-Lorentz equation for the impurity particle distribution $f_{0}\left(\mathbf{v}_{0}, t\right)$, as a functional of the fluid particle distribution, is the focus of this section.

An expansion of the impurity-fluid particle collision operator in powers of $h$ is straightforward, leading to the Kramers-Moyal representation [11]. The leading terms of this expansion have been given in Appendix A of reference [3],

$$
\begin{aligned}
\partial_{t} f_{0}\left(\mathbf{v}_{0}, t\right)= & \frac{\partial}{\partial \mathbf{v}_{0}} \cdot\left[h \mathbf{v}_{0} \gamma\left(v_{0}\right) f_{0}\left(\mathbf{v}_{0}, t\right)\right]+\frac{1}{2} \frac{\partial^{2}}{\partial v_{0 i} \partial v_{0 j}}\left\{h ^ { 2 } \left[n_{1}\left(v_{0}\right) \delta_{i j}\right.\right. \\
& \left.\left.+n_{2}\left(v_{0}\right)\left(v_{0 i} v_{0 j}-\frac{1}{3} \delta_{i j} v_{0}^{2}\right)\right] f_{0}\left(\mathbf{v}_{0}, t\right)\right\}+\mathcal{O}\left(h^{3}\right) .
\end{aligned}
$$

The friction $\gamma\left(v_{0}\right)$ and the noise functions $n_{1}\left(v_{0}\right), n_{2}\left(v_{0}\right)$ are explicit averages over the fluid particle distribution given in Appendix B. The states of interest are functions only of the magnitude of $\mathbf{v}_{0}$. Consequently, it is possible to introduce a variable

$$
\phi=\frac{v_{0}^{2}}{\left\langle v^{2}(t)\right\rangle}
$$

whose average value is the order parameter $\bar{\phi}(s)$, where $s$ is the dimensionless time variable defined in Eq. (5). The distribution function for this variable is $P(\phi, s)$, defined by

$$
P(\phi, s) \equiv 4 \pi f_{0}\left(v_{0}, t\right) v_{0}^{2} \frac{d v_{0}}{d \phi}=2 \pi\left\langle v^{2}(t)\right\rangle^{3 / 2} \phi^{1 / 2} f_{0}\left(v_{0}, t\right) .
$$

Then the Kramers-Moyal expansion becomes for $P(\phi, s)$

$$
\begin{aligned}
\partial_{s} P(\phi, s)= & \frac{\partial}{\partial \phi}\left\{\phi\left[-\xi^{*}+\gamma^{*}(\phi)\right]-\left(1-\frac{2}{3} \frac{\partial}{\partial \phi} \phi\right) h n_{1}^{*}(\phi)+\frac{4}{5} \frac{\partial}{\partial \phi} \phi^{2} h n_{2}^{*}(\phi)\right\} P(\phi, s) \\
& +\mathcal{O}\left(h^{2}\right) .
\end{aligned}
$$

Here $\xi^{*}$ is the dimensionless cooling rate for the fluid introduced in (2). The functions $\gamma^{*}(\phi), n_{1}^{*}(\phi)$, and $n_{2}^{*}(\phi)$ are the dimensionless forms of $\gamma\left(v_{0}\right), n_{1}\left(v_{0}\right)$, and $n_{2}\left(v_{0}\right)$, respectively, given by Eqs. (A27) and (B3) of the appendices [12]. They are functionals of the distribution function $f(v, t)$ of the fluid in the $\mathrm{HCS}$ and are normalized to have 
$\gamma^{*}(0)=1, n_{1}^{*}(0)=3\left\langle v^{3}\right\rangle / 4\left\langle v^{2}\right\rangle\langle v\rangle$, and $n_{2}^{*}(0)=1$. In addition, the derivative $\gamma^{* \prime}(\phi) \equiv d \gamma^{*}(\phi) / d \phi$ at $\phi=0$ is $\gamma^{* \prime}(0)=\left\langle v^{-1}\right\rangle\left\langle v^{2}\right\rangle / 5\langle v\rangle$. These functions can be accurately estimated by assuming a maximum entropy ensemble for the fluid $f$ and the results are given by Eqs. (B8)-(B10). According to these estimates, $n_{1}^{*}(0) \simeq 1$ and $\gamma^{* \prime}(0) \simeq 3 / 10$. Figure 8 shows the friction coefficient $\gamma^{*}(\phi)$ and the noise coefficients $n_{1}^{*}(\phi), n_{2}^{*}(\phi)$ according to this maximum entropy approximation for $f$. Hereafter, all the plots of quantities defined in terms of those coefficients will be made using Eqs. (B8)-(B10).

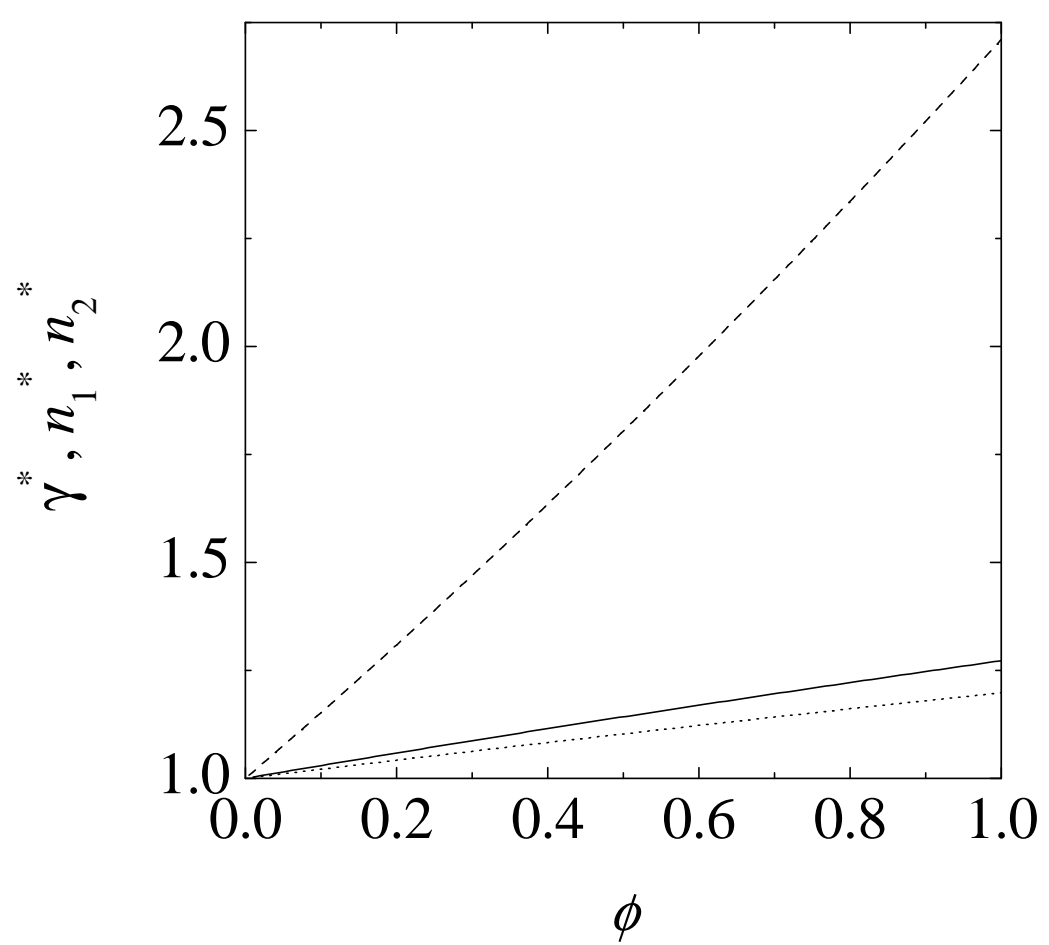

FIG. 8. Plot of the friction coefficient $\gamma^{*}(\phi)$ (solid line) and the noise functions $n_{1}^{*}(\phi)$ (dashed line) and $n_{2}^{*}(\phi)($ dotted line).

The Kramers-Moyal expansion is not well-ordered since the small parameter $h$ also multiplies the highest $\phi$ derivative. A proper asymptotic result requires a scaling such that all higher terms in the series are exactly zero in the appropriate limit. The simplest case is the deterministic limit for which $h=0$ in (47).

\section{A. Deterministic limit}

If the formal limit $h=0$ is taken in (47), the equation becomes

$$
\partial_{s} P_{0}(\phi, s)=\frac{\partial}{\partial \phi} \phi\left[-\xi^{*}+\gamma^{*}(\phi)\right] P_{0}(\phi, s)
$$

where the subindex 0 is used to denote quantities at $h=0$. The solution to this equation for sharp initial conditions $P_{0}(\phi, s=0)=\delta\left(\phi-\phi_{0}\right)$ is

$$
P_{0}(\phi, s)=\delta\left(\phi-\bar{\phi}_{0}(s)\right)
$$

with

$$
\left[\partial_{s}+\gamma^{*}\left(\bar{\phi}_{0}\right)-\xi^{*}\right] \bar{\phi}_{0}(s)=0
$$

and $\bar{\phi}_{0}(s=0)=\phi_{0}$. Thus the initial sharp distribution remains sharp and only its central value changes in time The latter defines the macroscopic dynamics for the average value $\bar{\phi}_{0}(s)$. As expected, it has the form (7) with a vanishing noise. The solution for more general initial conditions can be obtained as a superposition of the specific solution (49). 
The stationary solutions are obtained from 50 as the solution to

$$
\bar{\phi}_{0 \mathrm{~s}}\left[-\xi^{*}+\gamma^{*}\left(\bar{\phi}_{0 \mathrm{~s}}\right)\right]=0
$$

The possibilities are $\bar{\phi}_{0 \mathrm{~s}}=0$ and $\gamma^{*}\left(\bar{\phi}_{0 \mathrm{~s}}\right)=\xi^{*}$. It is shown in Appendix B that $\gamma^{*}(0)=1$. Therefore, the solution $\bar{\phi}_{0 \mathrm{~s}}=0$ is stable only if $\xi^{*}<1$. In the case $\xi^{*}>1$ the unique stable solution is determined from $\gamma^{*}\left(\bar{\phi}_{0 \mathrm{~s}}\right)=\xi^{*}$ with a non-zero value of $\bar{\phi}_{0 \mathrm{~s}}$. Such solutions exist because $\gamma^{*}(\bar{\phi})$ is a monotonically increasing function, i.e., $\gamma^{*}(\bar{\phi}) \geq \gamma^{*}(\overline{0})$, $\gamma^{* \prime}(\bar{\phi}) \geq 0$, as proved in Appendix B. These are the two phases discussed in Section III, now identified precisely from the Enskog-Lorentz kinetic equation. The details of the "equation of state" are different for this controlled analysis, but the qualitative features of states with $\bar{\phi}_{\mathrm{s}}=0$ and $\bar{\phi}_{\mathrm{s}} \neq 0$ for $h=0$ are recovered exactly, as illustrated in Fig. 9 .

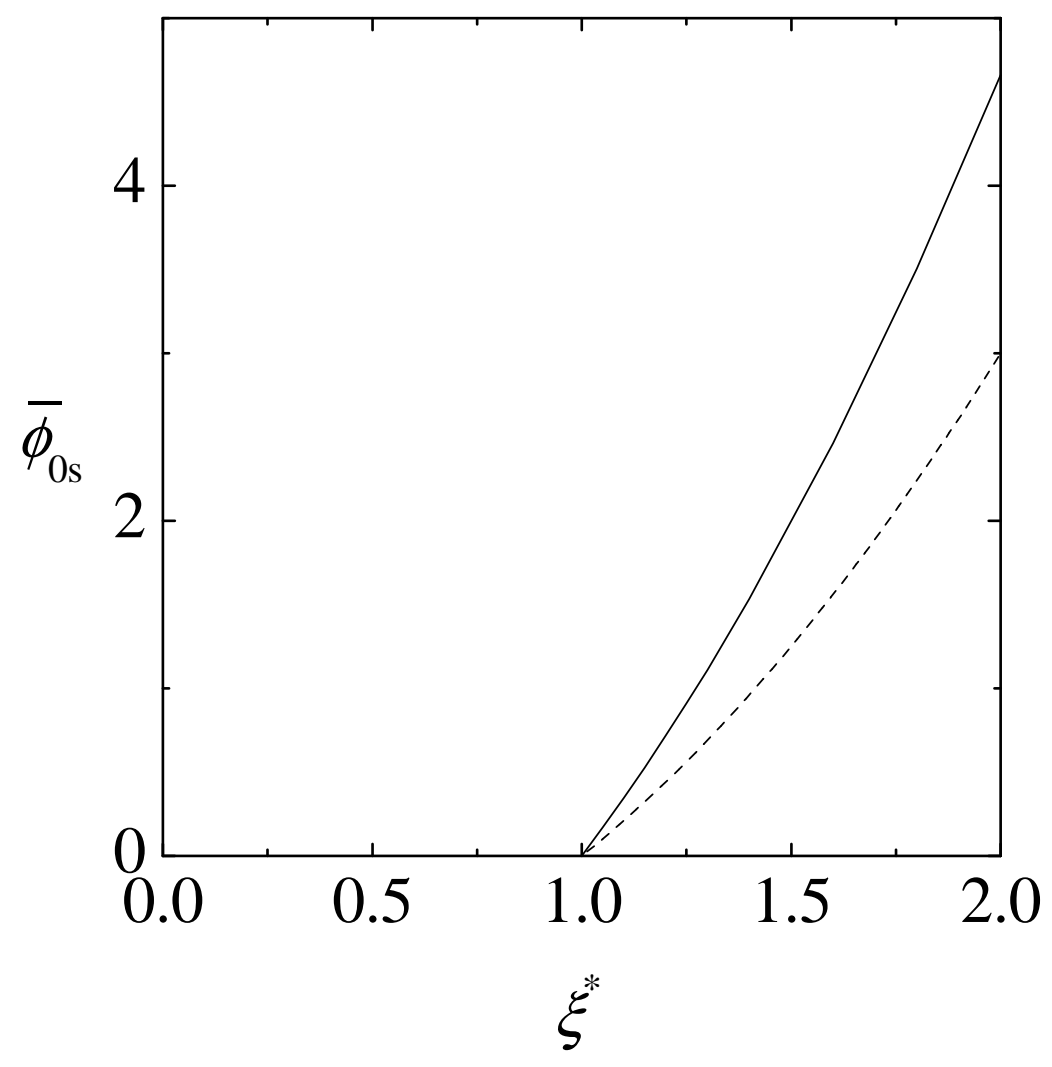

FIG. 9. Plot of the order parameter in the deterministic limit, $\bar{\phi}_{0 \mathrm{~s}}$, as a function of $\xi^{*}$. The dashed line is the maximum entropy estimate $\bar{\phi}_{\mathrm{s}}=\xi^{* 2}-1$ of Sec. II.

\section{B. Effects of fluctuations}

A more complete description including fluctuations is obtained by a transformation of the form $\phi=\bar{\phi}_{0}(s)+h^{p} \eta$, where $\bar{\phi}_{0}(s)$ is the average value of $\phi$ at $h=0$ and $\eta$ represents the fluctuations about this value. The power law of the scaling for the fluctuations is determined by the requirement that the distribution of fluctuations $\mathcal{P}(\eta, s, h)=$ $h^{p} P(\phi, s, h) \rightarrow \mathcal{P}(\eta, s)$ which is independent of $h$. Inverting the result in terms of $\phi$ gives the well-defined asymptotic behavior for small $h$ 11. Here, attention is limited to states near the stationary state $\bar{\phi}_{0 \text { s }}$ so the chosen scaling is $\phi=\bar{\phi}_{0 \mathrm{~s}}+h^{p} \eta$. The distribution function is no longer sharp, as in (49), but instead has a width proportional to $h^{p}$. The choice of $p$ is governed by the requirement that the Kramers-Moyal equation for the distribution of $\eta$ should truncate exactly for $h=0$. The details are given in Appendix B, where the stationary solution in the normal phase is found to be

$$
P_{\mathrm{s}}(\phi)=\frac{3}{h \bar{\eta}_{\mathrm{s}}}\left(\frac{3 \phi}{2 h \bar{\eta}_{\mathrm{s}} \pi}\right)^{1 / 2} e^{-3 \phi / 2 h \bar{\eta}_{\mathrm{s}}}, \quad \xi^{*}<1
$$


and the width of the distribution is characterized by

$$
\bar{\eta}_{\mathrm{s}}=\frac{n_{1}^{*}(0)}{1-\xi^{*}}=\frac{3\left\langle v^{3}\right\rangle}{4\left\langle v^{2}\right\rangle\langle v\rangle} \frac{1}{1-\xi^{*}} .
$$

Since $\bar{\phi}_{0 \mathrm{~s}}=0$ in this phase, the order parameter is $\bar{\phi}_{\mathrm{s}}=h \bar{\eta}_{\mathrm{s}}$. This agrees with the result of reference [3], where the distribution is recognized as a Maxwell-Boltzmann distribution for the velocity of the impurity particle, but with a different temperature than that of the fluid. In the present notation the impurity temperature identified from this Maxwellian is

$$
T_{0}=T \frac{1+\alpha_{0}}{2} \bar{\eta}_{\mathrm{s}}
$$

where $T$ is the granular temperature of the fluid. The phase transition is seen to occur with a diverging kinetic temperature for the impurity particle. If a maximum entropy distribution is assumed for the fluid, then the right side of (53) can be evaluated to get $\bar{\phi}_{\mathrm{s}}=h /\left(1-\xi^{*}\right)$, which agrees with the phenomenological theory of Section [1], Eq. (12).

In the ordered phase a qualitatively different distribution is obtained, as expected. It is now Gaussian in $\phi$ (quartic in velocity) and centered about a non-zero value

$$
P_{\mathrm{s}}(\phi)=\frac{1}{\sqrt{2 B\left(\bar{\phi}_{\mathrm{s}}\right) h \pi}} e^{-\left(\phi-\bar{\phi}_{\mathrm{s}}\right)^{2} / 2 B\left(\bar{\phi}_{\mathrm{s}}\right) h}, \quad \xi^{*}>1,
$$

with

$$
B(\phi)=\frac{1}{\gamma^{* \prime}(\phi)}\left[\frac{2}{3} n_{1}^{*}(\phi)+\frac{4}{5} \phi n_{2}^{*}(\phi)\right]
$$

The function $B(\phi)$ is plotted in Fig. 10. The width of the distribution is $\Delta \phi_{\mathrm{s}}=\left[B\left(\bar{\phi}_{\mathrm{s}}\right) h\right]^{1 / 2}$, so that as $h \rightarrow 0$ the distribution becomes sharply peaked about the stationary value $\bar{\phi}_{\mathrm{s}}=\bar{\phi}_{0 \mathrm{~s}}$, where $\bar{\phi}_{0 \mathrm{~s}}$ is the stationary order parameter in the deterministic limit. At a fixed small value of $h$ the (absolute) width $\Delta \phi_{\mathrm{s}}$ increases, but the relative width $\Delta \phi_{\mathrm{s}} / \bar{\phi}_{\mathrm{s}}$ decreases, as $\xi^{*}-1$ (and, consequently, $\bar{\phi}_{\mathrm{s}}$ ) increases. 


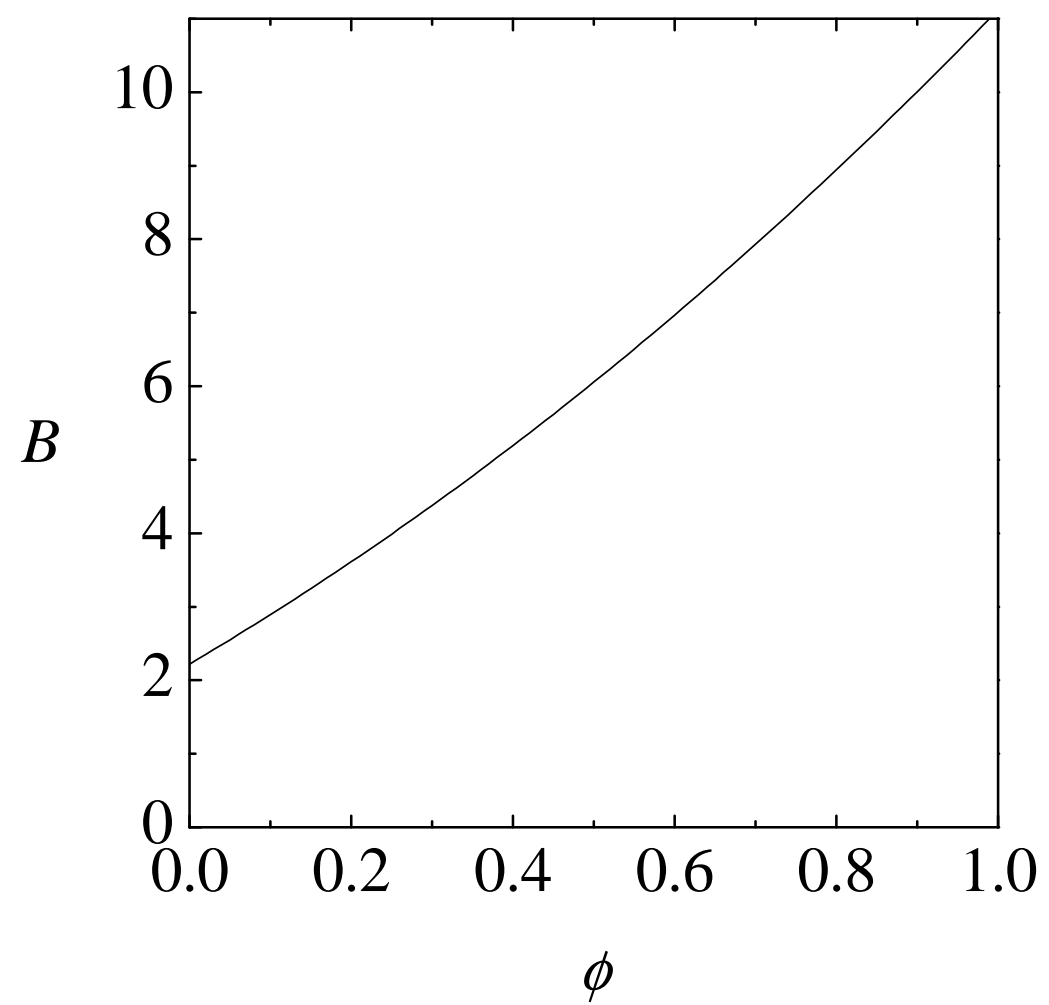

FIG. 10. Plot of the function $B(\phi)$ defined in Eq. (56).

\section{Critical domain}

The above results distinguish the cases of $h \rightarrow 0$ for $\xi^{*}<1$ and for $\xi^{*}>1$. A uniform description of the critical domain for small $h$ and $\xi^{*} \approx 1$ can be obtained by noting that $\bar{\phi}_{\mathrm{s}}$ vanishes at the critical point from both phases, and scaling the Kramers-Moyal equation according to $\xi^{*}-1=h^{1 / 2} \delta$ and $\phi=h^{1 / 2} \eta$. In addition a new time variable is defined by $\tau=h^{1 / 2} s$. Then at $h=0$ the equation is

$$
\partial_{\tau} \mathcal{P}(\eta, \tau)=\frac{\partial}{\partial \eta}\left[-\delta \eta+\gamma^{* \prime}(0) \eta^{2}-\frac{1}{3}\left(3-2 \frac{\partial}{\partial \eta} \eta\right) n_{1}^{*}(0)\right] \mathcal{P}(\eta, \tau) .
$$

The stationary distribution function is found to be

$$
\mathcal{P}_{\mathrm{S}}(\eta)=C \eta^{1 / 2} \exp \left[-\frac{1}{2 B(0)}\left(\eta-\frac{\delta}{\gamma^{* \prime}(0)}\right)^{2}\right],
$$

where $B(0)=2 n_{1}^{*}(0) / 3 \gamma^{* \prime}(0) \simeq 2.22$ is the value at $\phi=0$ of the function defined in Eq. (56). The scaled order parameter in this critical domain is then obtained from

$$
\bar{\eta}_{\mathrm{s}}(\delta)=\sqrt{B(0)} \frac{\int_{0}^{\infty} d u u^{3 / 2} \exp \left[-(u-2 z)^{2} / 2\right]}{\int_{0}^{\infty} d u u^{1 / 2} \exp \left[-(u-2 z)^{2} / 2\right]},
$$

where $z \equiv \delta / 2 \gamma^{* \prime}(0) \sqrt{B(0)}$. Its explicit expression is

$$
\bar{\eta}_{\mathrm{s}}(\delta)=\sqrt{B(0)} \frac{\Psi_{1}(z)}{4 z \Psi_{2}(z)}
$$

where 


$$
\begin{aligned}
& \Psi_{1}(z)= \begin{cases}\left(3+4 z^{2}\right) \Psi_{2}(z)+2 z^{2}\left[K_{7 / 4}\left(z^{2}\right)+K_{-1 / 4}\left(z^{2}\right)-K_{5 / 4}\left(z^{2}\right)-K_{-3 / 4}\left(z^{2}\right)\right], & z<0 \\
\left(3+6 z^{2}\right) \Psi_{2}(z)+2 z^{2}\left[I_{7 / 4}\left(z^{2}\right)+I_{-7 / 4}\left(z^{2}\right)+I_{5 / 4}\left(z^{2}\right)+I_{-5 / 4}\left(z^{2}\right)\right], & z>0,\end{cases} \\
& \Psi_{2}(z)= \begin{cases}K_{1 / 4}\left(z^{2}\right)-K_{3 / 4}\left(z^{2}\right), & z<0, \\
I_{3 / 4}\left(z^{2}\right)+I_{-3 / 4}\left(z^{2}\right)+I_{1 / 4}\left(z^{2}\right)+I_{-1 / 4}\left(z^{2}\right), & z>0,\end{cases}
\end{aligned}
$$

$I_{\nu}(z)$ and $K_{\nu}(z)$ being the modified Bessel functions of first and second kind, respectively. The width of the distribution $\Delta \eta=\sqrt{\overline{\eta^{2}}-\bar{\eta}^{2}}$ in the steady state can be obtained by taking moments in Eq. (57) as

$$
\Delta \eta_{\mathrm{s}}(\delta)=\left[\frac{n_{1}^{*}(0)+\delta \bar{\eta}_{\mathrm{s}}(\delta)}{\gamma^{* \prime}(0)}-\bar{\eta}_{\mathrm{s}}^{2}(\delta)\right]^{1 / 2}
$$

The asymptotic behaviors of $\bar{\eta}_{\mathrm{s}}(\delta)$ and $\Delta \eta_{\mathrm{s}}(\delta)$ are

$$
\begin{gathered}
\bar{\eta}_{\mathrm{s}}(\delta) \rightarrow \begin{cases}n_{1}^{*}(0)|\delta|^{-1}, & \delta \rightarrow-\infty, \\
\sqrt{\lambda_{1}}, & \delta \rightarrow 0, \\
\delta / \gamma^{* \prime}(0), & \delta \rightarrow \infty,\end{cases} \\
\Delta \eta_{\mathrm{s}}(\delta) \rightarrow \begin{cases}\sqrt{2 / 3} n_{1}^{*}(0)|\delta|^{-1}, & \delta \rightarrow-\infty, \\
\sqrt{\lambda_{2}}, & \delta \rightarrow 0, \\
\sqrt{B(0)}, & \delta \rightarrow \infty,\end{cases}
\end{gathered}
$$

where $\lambda_{1} \equiv 2 B(0)[\Gamma(5 / 4) / \Gamma(3 / 4)]^{2} \simeq 2.43$ and $\lambda_{2} \equiv 3 B(0) / 2-\lambda_{1} \simeq 0.90$. In the limit $\delta \rightarrow-\infty$ we have $\Delta \eta_{\mathrm{s}} / \bar{\eta}_{\mathrm{s}} \rightarrow$ $\sqrt{2 / 3}$, which is consistent with a Maxwell-Boltzmann distribution. In contrast, $\Delta \eta_{\mathrm{s}} / \bar{\eta}_{\mathrm{s}} \rightarrow 0$ when $\delta \rightarrow \infty$, so that the distribution is sharp around the order parameter in that limit. The dependence of the scaled order parameter $\bar{\eta}_{\mathrm{s}}(\delta)$ on the scaled control parameter $\delta$ is shown in Fig. 11, where also the width of the distribution is plotted.

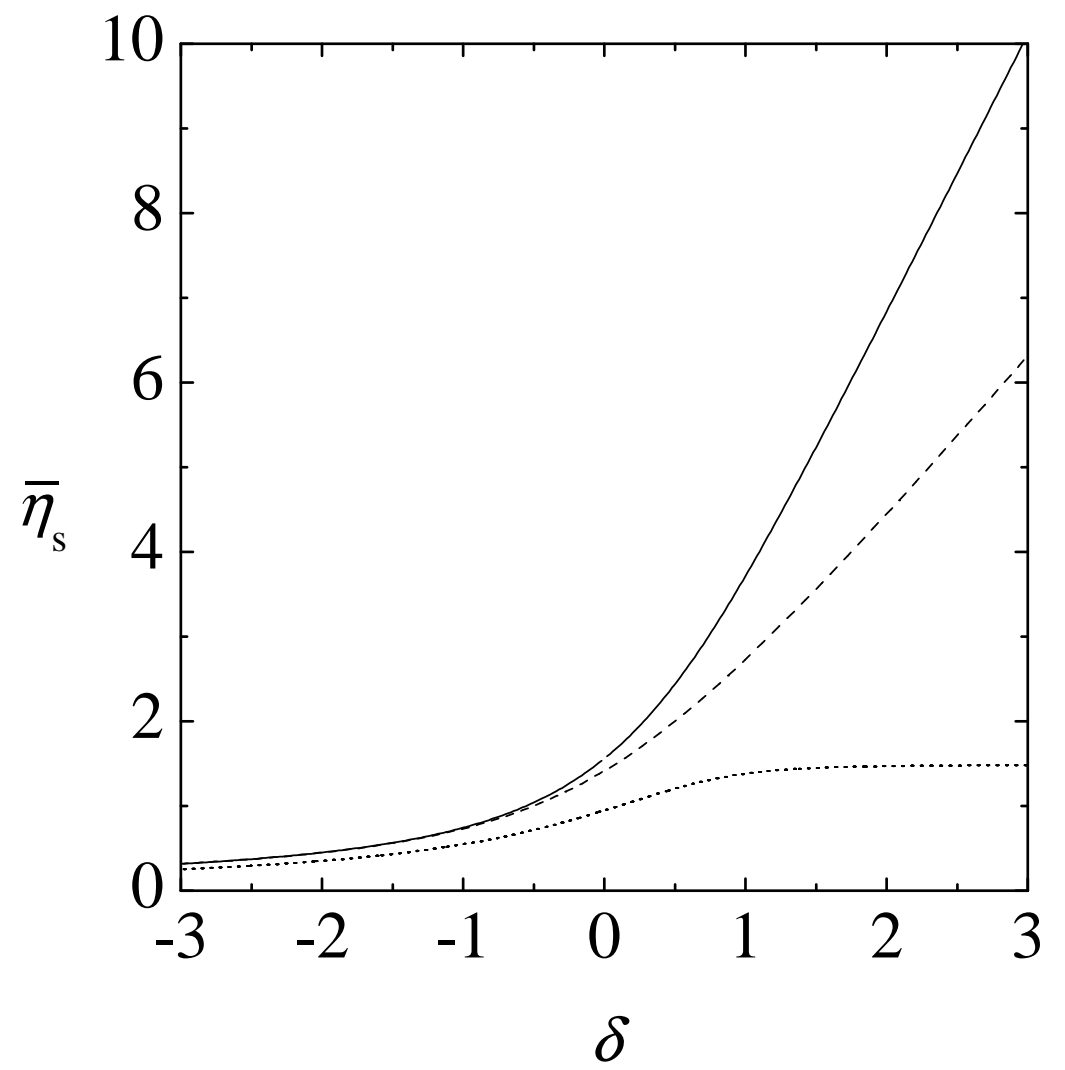


FIG. 11. Plot of $\bar{\eta}_{\mathrm{s}}(\delta)$ (solid line) and $\Delta \eta_{\mathrm{s}}$ (dotted line). The dashed line represents the phenomenological approximation $\bar{\eta}_{\mathrm{s}}(\delta) \rightarrow \delta+\sqrt{\delta^{2}+2}$, Eq. (14).

It is worth noting that the scaling relations $\phi=h^{1 / 2} \eta, \xi^{*}-1=h^{1 / 2} \delta$ are successfully captured by the phenomenological theory of Sec. II, except that there the scaling function is approximated by $\bar{\eta}_{\mathrm{s}}(\delta) \rightarrow \delta+\sqrt{\delta^{2}+2}$, Eq. $(14)$. While this function is not quantitatively correct, especially for $\delta>0$ (cf. Fig. 11), it is qualitatively consistent with the limits in (64), the numerical coefficients being replaced by $\lambda_{1} \simeq 2.43 \rightarrow 2, \gamma^{* \prime}(0) \simeq 0.3 \rightarrow \bar{\gamma}^{* \prime}(0)=1 / 2$.

Let us now go back to the unscaled variable $\phi$. The corresponding distribution in the critical domain is

$$
P_{\mathrm{S}}(\phi) \propto \phi^{1 / 2} \exp \left[-\frac{1}{2 B(0) h}\left(\phi-\frac{\xi^{*}-1}{\gamma^{* \prime}(0)}\right)^{2}\right]
$$

and the equation of state is

$$
\bar{\phi}_{\mathrm{s}}\left(\xi^{*}, h\right)=h^{1 / 2} \bar{\eta}_{\mathrm{s}}\left(\frac{\xi^{*}-1}{h^{1 / 2}}\right) .
$$

This result encompasses the normal and ordered phases, as well as the critical point. The normal phase in the critical domain is defined by $h \ll 1-\xi^{*}$ (i.e., $\left.\delta \rightarrow-\infty\right)$, the ordered phase is recovered in the case $h \ll \xi^{*}-1$ (i.e., $\delta \rightarrow \infty$ ), while the critical point corresponds to $\xi^{*}=1(\delta=0)$. Thus, the asymptotic behaviors (64) translate into

$$
\lim _{h \rightarrow 0} \bar{\phi}_{\mathrm{s}}\left(\xi^{*}, h\right)= \begin{cases}h n_{1}^{*}(0) /\left(1-\xi^{*}\right), & \xi^{*} \lesssim 1 \\ \sqrt{\lambda_{1} h}, & \xi^{*}=1 \\ \left(\xi^{*}-1\right) / \gamma^{* \prime}(0), & \xi^{*} \gtrsim 1\end{cases}
$$

In the normal phase, $\bar{\phi}_{\mathrm{s}} \sim h \ll 1-\xi^{*}$, so that the distribution (66) becomes

$$
P_{\mathrm{S}}(\phi) \propto \phi^{1 / 2} \exp \left[-\frac{3 \phi\left(1-\xi^{*}\right)}{2 h n_{1}^{*}(0)}\right],
$$

which agrees with (52). In the ordered phase, however, the width of the distribution is much smaller than the average value $\bar{\phi}_{\mathrm{s}}=\left(\xi^{*}-1\right) / \gamma^{* \prime}(0)$ [which is the solution to $\xi^{*}=\gamma^{*}\left(\bar{\phi}_{\mathrm{s}}\right)$ in the critical region], so that the prefactor $\phi^{1 / 2}$ in (66) can be replaced by a $\bar{\phi}_{\mathrm{s}}^{1 / 2}$ with the result

$$
P_{\mathrm{s}}(\phi) \propto e^{-\left(\phi-\bar{\phi}_{\mathrm{s}}\right)^{2} / 2 B(0) h} .
$$

As expected, (70) agrees with (55) particularized to the critical region. Finally, at the critical point the distribution is

$$
P_{\mathrm{S}}(\phi) \propto \phi^{1 / 2} e^{-\phi^{2} / 2 B(0) h}
$$

As anticipated from the behavior of $\Delta \eta_{\mathrm{s}} / \bar{\eta}_{\mathrm{s}}$, there exists a crossover in the critical domain from the MaxwellBoltzmann distribution (69) to the sharp distribution (70) through (71). Of course, the distribution function (66) is more general than the three limiting cases described by (69)-(71). To focus on the shape of the distribution function around its average value, define the normalized distribution $P_{\mathrm{s}}^{*}(x)=\bar{\phi}_{\mathrm{s}} P_{\mathrm{s}}\left(\phi=x \bar{\phi}_{\mathrm{s}}\right)=\bar{\eta}_{\mathrm{s}} \mathcal{P}_{\mathrm{s}}\left(\eta=x \bar{\eta}_{\mathrm{s}}\right)$. From Eq. $(58)$ we have

$$
P_{\mathrm{s}}^{*}(x) \propto x^{1 / 2} \exp \left[-\frac{1}{2}\left(x \frac{\bar{\eta}_{\mathrm{s}}}{\sqrt{B(0)}}-2 z\right)^{2}\right], \quad z \equiv \delta / 2 \gamma^{* \prime}(0) \sqrt{B(0)}
$$

By construction, this distribution is normalized to $\langle x\rangle=1$, regardless of the value of the scaled control parameter $\delta$. The asymptotic forms of $P_{\mathrm{s}}^{*}(x)$ are

$$
P_{\mathrm{s}}^{*}(x) \rightarrow \begin{cases}3 \sqrt{\frac{3 x}{2 \pi}} e^{-3 x / 2}, & \delta \rightarrow-\infty, \\ 2 \frac{[\Gamma(5 / 4)]^{3 / 2}}{[\Gamma(3 / 4)]^{5 / 2}} x^{1 / 2} \exp \left\{-\left[\frac{\Gamma(5 / 4)}{\Gamma(3 / 4)} x^{2}\right\},\right. & \delta \rightarrow 0, \\ \sqrt{\frac{2}{\pi}} z \exp \left[-2 z^{2}(x-1)^{2}\right], & \delta \rightarrow \infty .\end{cases}
$$


The crossover of the normalized distribution $P_{\mathrm{s}}^{*}(x)$ from the Maxwell-Boltzmann form corresponding to $\delta \rightarrow-\infty$ to the sharp distribution corresponding to $\delta=5$ is illustrated in Fig. 12.

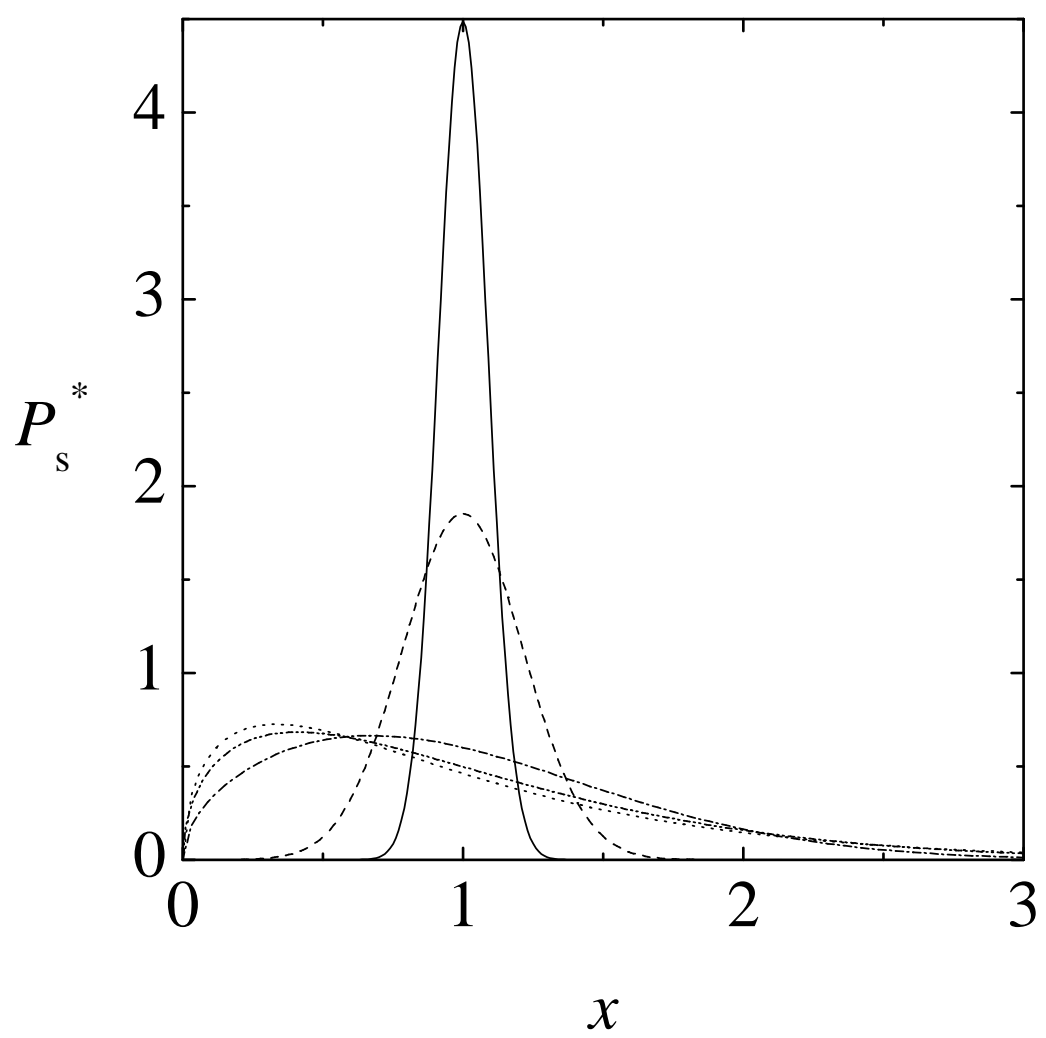

FIG. 12. Plot of the normalized distribution function $P_{\mathrm{s}}^{*}(x)$ for $\delta \equiv\left(\xi^{*}-1\right) / h^{1 / 2}=-\infty(\cdots),-1(-\cdot \cdot-), 0(-\cdot-), 2(--)$, and $5(-)$.

\section{Critical dynamics}

To study the dynamics in the critical domain define a deviation from the stationary solution by

$$
\mathcal{P}(\eta, \tau)=\mathcal{P}_{\mathbf{s}}(\eta)[1+y(\eta, \tau)]
$$

Equation (57) becomes

$$
\partial_{\tau} y(\eta, \tau)=\frac{2}{3} n_{1}^{*}(0) \frac{1}{\mathcal{P}_{\mathrm{s}}(\eta)} \frac{\partial}{\partial \eta} \mathcal{P}_{\mathrm{s}}(\eta) \eta \frac{\partial}{\partial \eta} y(\eta, \tau)
$$

Now we define a $\mathcal{F}[y]$ by

$$
\mathcal{F}[y]=\int_{0}^{\infty} d \eta \mathcal{P}_{\mathrm{s}}(\eta)[y(\eta, \tau)]^{2}
$$

Then, from Eq. (75) we have

$$
\partial_{\tau} \mathcal{F}[y]=-\frac{4}{3} n_{1}^{*}(0) \int_{0}^{\infty} d \eta \mathcal{P}_{\mathbf{s}}(\eta) \eta\left[\frac{\partial}{\partial \eta} y(\eta, \tau)\right]^{2}
$$

Thus, $\mathcal{F}[y]$ has the required properties of a Lyapunov functional for the dynamics of $y(\eta, \tau)$, namely 13] 


$$
\mathcal{F}[y] \geq 0, \quad \partial_{\tau} \mathcal{F}[y] \leq 0,
$$

the equality being verified for $y=0$ only. This implies that for any initial condition the solution to (75) evolves in time towards $y(\eta, \tau) \rightarrow 0$.

The dynamics for $\bar{\eta}(\tau)$ is given by

$$
\left(\partial_{\tau}-\delta\right) \bar{\eta}+\gamma^{* \prime}(0) \overline{\eta^{2}}=n_{1}^{*}(0) .
$$

This is not a closed equation so in principle it is necessary first to solve (57) for the distribution function and then calculate $\bar{\eta}$. However, an estimate can be obtained from (79) using the approximation $\overline{\eta^{2}} \sim\left(\bar{\eta}^{2}{ }_{\mathrm{s}} / \bar{\eta}_{\mathrm{s}}^{2}\right) \bar{\eta}^{2}$. Then the linearized equation for $x=\bar{\eta}-\bar{\eta}_{\mathrm{s}}$ obtained from (79) for small $x$ is

$$
\partial_{\tau} x+\left[\delta+\frac{2 n_{1}^{*}(0)}{\bar{\eta}_{\mathrm{s}}}\right] x=0 .
$$

Of course, $\delta+2 n_{1}^{*}(0) / \bar{\eta}_{\mathrm{s}}>0$ for $\delta \geq 0$. It can be verified from Eqs. (61) and (62) that $\Psi_{1}(z) / \Psi_{2}(z) \geq-3$ for $z<0$, so that $\delta+2 n_{1}^{*}(0) / \bar{\eta}_{\mathrm{s}} \geq|\delta|$ for $\delta<0$. This confirms the above stability analysis. Equation (80) is consistent with $(\mathrm{B} 18)$ for the ordered phase and $(\overline{\mathrm{B} 23})$ for the normal phase. The finite relaxation time $\bar{\eta}_{\mathrm{s}} / 2 n_{1}^{*}(0)$ at the critical point is not in conflict with the divergent relaxation time in (31) since the unit of time is different (i.e., $\tau=\sqrt{h} s$ ).

\section{DISCUSSION}

In detail application of statistical mechanics methods to the model granular fluid of hard spheres with inelastic collisions exposes important differences from normal fluids. First among these is the replacement of the equilibrium Gibbs state with the time dependent homogeneous cooling state. In the case of mixtures, the absence of detailed balance in collisions leads to a breakdownwn of the usual equipartition theorem for normal fluids. This is interesting (e.g., the HCS for a binary mixture has two kinetic temperatures) but is perhaps not too surprising. In the case of a single, mechanically different, impurity particle in a one component granular fluid this effect is easily understood as a competition between the average impurity-fluid collision rate $\nu_{c}$, responsible for "equilibration," and the cooling rate for the fluid $\xi$ constantly changing the reference state. This competition is most severe for conditions such that the average impurity-fluid collision rate decreases at constant $\xi$, as occurs when the impurity mass is much larger than that of the fluid particle. This requires that the nonlinear dependence of the actual impurity-fluid collision rate on impurity mean square velocity is activated to increase the true collision rate. As a consequence, the joint HCS for the fluid and impurity is maintained but with a much higher speed for the impurity relative to that for the fluid particles. In the limit of infinite impurity mass an extreme breakdown of equipartition occurs with the single impurity particle attaining a finite fraction of the total kinetic energy.

This peculiar feature distinguishes the conditions of $\xi / \nu_{c}=\xi^{*}<1$, where the distribution of energies is similar to that for a normal fluid, from $\xi^{*}>1$ where the distribution is anomalous. A surprising feature of the description given here for these two cases is the exact analogy to a second order phase transition in a normal fluid. The order parameter is the ratio of impurity to fluid particle mean square velocities, $\bar{\phi}_{\mathrm{s}}$, the conjugate field is $h$ (a measure of the mass ratio), and the role of the inverse temperature is the relative cooling rate $\xi^{*}$. To summarize the primary results obtained here the following comments are offered:

- The nonlinearity of the impurity-fluid particle collision rate, expressed by the dimensionless friction constant $\gamma^{*}(\phi)$, is essentially the same as that for an impurity in a normal fluid. In the latter case $\phi \ll 1$ for a heavy impurity particle and the relevant values are $\gamma^{*}(\phi) \approx \gamma^{*}(0)=1$. However, when the background fluid is cooling it is necessary that $\gamma^{*}(\phi) \approx \xi^{*}$ so values of $\phi$ of order 1 are selected when $\xi^{*}>1$. The details of the mechanism by which the host fluid cools is unimportant for this qualitative effect. In fact, even if all collisions are elastic, the same two phases would occur if the fluid were cooled by an external thermostat.

- The thermodynamic analogy originates from an "equation of state" $h=h\left(\bar{\phi}_{\mathrm{s}}, \xi^{*}\right)$, obtained from the "equilibration" condition for the HCS. The phenomenological estimate in Section III and the exact asymptotic kinetic theory analysis of Section IV] are essentially the same. The "Gibbs free energy" obtained from integrating the equation of state has a Landau-like form near the critical point with critical exponents associated with the various first and second derivatives. In particular, the susceptibility diverges, indicating a second order phase transition. 
- The approach to the HCS is stable in both phases. The dynamics is governed by a Ginzburg-Landau equation defined in terms of the Gibbs free energy. Near the phase transition there is critical slowing, with the characteristic relaxation time diverging proportional to the susceptibility. Alternatively this can be viewed as a change of time scale from $s$ to $\tau=\sqrt{h} s$.

- The diffusion coefficient is finite in the normal phase but diverges on approaching the transition. It remains divergent in the ordered phase. In terms of the velocity autocorrelation function this is seen to be a divergent relaxation time for the decay of correlations, and consequently, the mean square displacement is characterized by ballistic rather than diffusive dynamics.

- The HCS velocity distribution in both phases and in the critical region is obtained from an exact asymptotic analysis of the Enskog-Lorentz equation. In the normal phase away from the critical point it is a Maxwellian with a temperature different from that of the fluid. In the ordered phase it is a quartic function of the velocity centered about a non-zero average speed. The distribution function in the critical region exhibits a continuous crossover between these distributions as the cooling rate changes from $\xi^{*} \leq 1$ to $\xi^{*} \geq 1$.

The most direct and controlled observation of the phenomena described here would be via Monte Carlo simulation of the Boltzmann-Lorentz equation or molecular dynamics simulation. The qualitative change in the distribution function for the ordered phase already has been seen in Monte Carlo simulation, Fig. 6 of Ref. 狛. In principle, the Monte Carlo simulation could provide access to the longer time behavior associated with critical dynamics and diffusion near the critical point. Experimental conditions for real fluids are more difficult to imagine, since a cooling medium for the impurity particle is required. However, as noted above, the cooling does not have to be associated with inelastic collisions. Thus an impurity particle in a continuously and homogeneously quenched fluid should exhibit the same phase transition.

The extreme breakdown of equipartition discussed in this paper extends to the case of a mixture as well, where a mole fraction $x_{0}$ of impurity particles exists instead of just one impurity particle. In that case a phenomenological description similar to that of Sec. II shows that the critical value of the control parameter $\xi^{*}$ in the limit $h \rightarrow 0$ at finite $x_{0} / h$ is $\xi_{c}^{*}=1-\left(x_{0} / h\right)\left(1-\alpha_{0}^{2}\right) / 4$, so that the HCS of the mixture is always in an ordered state $\left(\xi_{c}^{*}=0\right)$ if $x_{0} / h \geq 4 /\left(1-\alpha_{0}^{2}\right)$. The details of this case will be published elsewhere.

\section{ACKNOWLEDGMENTS}

This research was supported by National Science Foundation grant PHY 9722133. A.S. acknowledges partial support from the Ministerio de Ciencia y Tecnología (Spain) through grant No. BFM2001-0718 and through a sabbatical grant No. PR2000-0117.

\section{APPENDIX A: COOLING RATES AND COLLISION FREQUENCY}

The cooling rates $\xi$ and $\xi_{0}$ for a fluid and the impurity particle are defined by (2), while the diffusion coefficient in Section III is expressed in terms of a related frequency $\omega_{D}$. They can be written as

$$
\xi=-\frac{\partial_{t}\left\langle v^{2}(t)\right\rangle}{\left\langle v^{2}(t)\right\rangle}, \quad \xi_{0}=-\frac{\partial_{t}\left\langle v_{0}^{2}(t)\right\rangle}{\left\langle v_{0}^{2}(t)\right\rangle}, \quad \omega_{D}=-\frac{1}{2} \xi_{0}+\nu_{0},
$$

where the impurity-fluid particle collision frequency is

$$
\nu_{0}=-\left.\frac{\partial_{t^{\prime}}\left\langle\mathbf{v}_{0}(t) \cdot \mathbf{v}_{0}\left(t+t^{\prime}\right)\right\rangle}{\left\langle v_{0}^{2}(t)\right\rangle}\right|_{t^{\prime}=0} .
$$

The subscript 0 denotes the velocity for the impurity particle and the brackets denote an average over the initial ensemble. The time derivatives can be expressed in terms of the generator $L$ for the inelastic hard sphere dynamics [9, 14,

$$
\begin{gathered}
\partial_{t} X(t)=L X(t), \\
L=\mathbf{v}_{0} \cdot \nabla_{0}+\sum_{i=1}^{N} \mathbf{v}_{i} \cdot \nabla_{i}+\sum_{i=1}^{N} T(i, 0)+\frac{1}{2} \sum_{i=1}^{N} \sum_{j \neq i}^{N} T(i, j) .
\end{gathered}
$$


The binary collision operators for fluid-fluid and fluid-impurity pairs are defined by

$$
\begin{gathered}
T(i, j)=-\sigma^{2} \int d \Omega \Theta\left(-\mathbf{v}_{i j} \cdot \widehat{\boldsymbol{\sigma}}\right)\left(\mathbf{v}_{i j} \cdot \widehat{\boldsymbol{\sigma}}\right) \delta\left(\mathbf{r}_{i j}-\boldsymbol{\sigma}\right)\left(b_{i j}-1\right), \\
T(i, 0)=-\bar{\sigma}^{2} \int d \Omega \Theta\left(-\mathbf{v}_{i 0} \cdot \widehat{\boldsymbol{\sigma}}\right)\left(\mathbf{v}_{i 0} \cdot \widehat{\boldsymbol{\sigma}}\right) \delta\left(\mathbf{r}_{i 0}-\overline{\boldsymbol{\sigma}}\right)\left(b_{i 0}-1\right),
\end{gathered}
$$

where $\boldsymbol{\sigma}=\sigma \widehat{\boldsymbol{\sigma}}, \overline{\boldsymbol{\sigma}}=\bar{\sigma} \widehat{\boldsymbol{\sigma}}$, and $b_{i j}$ and $b_{i 0}$ transform the relative velocity for the pairs into their scattered velocities and leave the center of mass invariant,

$$
\begin{aligned}
b_{i j} \mathbf{v}_{i j} & =\mathbf{v}_{i j}-(1+\alpha)\left(\mathbf{v}_{i j} \cdot \widehat{\boldsymbol{\sigma}}\right) \widehat{\boldsymbol{\sigma}}, \quad b_{i j} \mathbf{G}_{i j}=\mathbf{G}_{i j}, \\
b_{i 0} \mathbf{v}_{i 0} & =\mathbf{v}_{i 0}-\left(1+\alpha_{0}\right)\left(\mathbf{v}_{i 0} \cdot \widehat{\boldsymbol{\sigma}}\right) \widehat{\boldsymbol{\sigma}}, \quad b_{i 0} \mathbf{G}_{i 0}=\mathbf{G}_{i 0} .
\end{aligned}
$$

The various velocities and reduced masses are given by

$$
\begin{gathered}
\mathbf{v}_{i 0}=\mathbf{v}_{i}-\mathbf{v}_{0}, \quad \mathbf{G}_{i 0}=\mu \mathbf{v}_{i}+\mu_{0} \mathbf{v}_{0}, \quad \mu=\frac{m}{m+m_{0}}, \quad \mu_{0}=\frac{m_{0}}{m+m_{0}}, \\
\mathbf{v}_{i j}=\mathbf{v}_{i}-\mathbf{v}_{j}, \quad \mathbf{G}_{i j}=\frac{1}{2}\left(\mathbf{v}_{i}+\mathbf{v}_{j}\right), \\
\mathbf{v}_{i}=\mathbf{G}_{i 0}+\mu_{0} \mathbf{v}_{i 0}, \quad \mathbf{v}_{0}=\mathbf{G}_{i 0}-\mu \mathbf{v}_{i 0}, \quad \mathbf{v}_{j}=\mathbf{G}_{i j}-\frac{1}{2} \mathbf{v}_{i j} .
\end{gathered}
$$

In terms of the binary collision operators the cooling rates and collision frequencies become

$$
\begin{gathered}
\xi=-\frac{(N-1)\left\langle T(2,1) v_{1}^{2}\right\rangle+\left\langle T(1,0) v_{1}^{2}\right\rangle}{\left\langle v^{2}\right\rangle}, \quad \xi_{0}=-N \frac{\left\langle T(1,0) v_{0}^{2}\right\rangle}{\left\langle v_{0}^{2}\right\rangle}, \\
\nu_{0}=-N \frac{\left\langle\mathbf{v}_{0} \cdot T(1,0) \mathbf{v}_{0}\right\rangle}{\left\langle v_{0}^{2}\right\rangle}
\end{gathered}
$$

In the following it is assumed that terms of relative order $1 / N$ can be neglected. Substitution of the definitions for $T(2,1)$ and $T(1,0)$ leads to

$$
\begin{gathered}
T(2,1) v_{1}^{2}=-\sigma^{2} \int d \Omega \Theta\left(-\mathbf{v}_{21} \cdot \widehat{\boldsymbol{\sigma}}\right)\left(\mathbf{v}_{21} \cdot \widehat{\boldsymbol{\sigma}}\right)^{2} \delta\left(\mathbf{r}_{21}-\boldsymbol{\sigma}\right)\left[(1+\alpha)\left(\mathbf{G}_{21} \cdot \widehat{\boldsymbol{\sigma}}\right)-\frac{1}{4}\left(1-\alpha^{2}\right)\left(\mathbf{v}_{21} \cdot \widehat{\boldsymbol{\sigma}}\right)\right], \\
T(1,0) v_{0}^{2}=-\bar{\sigma}^{2} 4 h \int d \Omega \Theta\left(-\mathbf{v}_{10} \cdot \widehat{\boldsymbol{\sigma}}\right)\left(\mathbf{v}_{10} \cdot \widehat{\boldsymbol{\sigma}}\right)^{2} \delta\left(\mathbf{r}_{10}-\overline{\boldsymbol{\sigma}}\right)\left(h \mathbf{g}_{10}+\mathbf{v}_{0}\right) \cdot \widehat{\boldsymbol{\sigma}} \\
\mathbf{v}_{0} \cdot T(1,0) \mathbf{v}_{0}=-\bar{\sigma}^{2} 2 h \int d \Omega \Theta\left(-\mathbf{v}_{10} \cdot \widehat{\boldsymbol{\sigma}}\right)\left(\mathbf{v}_{10} \cdot \widehat{\boldsymbol{\sigma}}\right)^{2} \delta\left(\mathbf{r}_{10}-\overline{\boldsymbol{\sigma}}\right) \mathbf{v}_{0} \cdot \widehat{\boldsymbol{\sigma}}
\end{gathered}
$$

Since these are all two-particle functions the averages in (A12) and $\mathrm{A} 13$ ) can be reduced to integrals over the two-particle reduced distribution functions $f^{(2)}$ and $f_{0}^{(2)}$ defined in terms of the $N$-particle distribution function $\rho_{\mathrm{s}}$ as

$$
\begin{gathered}
f^{(2)}\left(x_{1}, x_{2}\right)=V^{2} \int d x_{0} d x_{3} \ldots d x_{N} \rho_{\mathrm{s}}(\Gamma) \\
f_{0}^{(2)}\left(x_{0}, x_{1}\right)=V^{2} \int d x_{2} \ldots d x_{N} \rho_{\mathrm{s}}(\Gamma) .
\end{gathered}
$$


Here $V$ is the volume and $x_{i}$ denotes a point in the six-dimensional phase space of particle $i$, i.e $x_{i} \Leftrightarrow\left\{\mathbf{q}_{i}, \mathbf{v}_{i}\right\}$. The frequencies then become

$$
\begin{aligned}
\xi=\frac{1}{4} n \sigma^{2}\left(1-\alpha^{2}\right) v_{f} \int d \mathbf{v}_{1}^{*} d \mathbf{v}_{2}^{*} \int d \Omega f^{(2) *}\left(\mathbf{v}_{1}^{*}, \mathbf{v}_{2}^{*}, \mathbf{r}_{21}=-\boldsymbol{\sigma}\right) \Theta\left(\mathbf{v}_{21}^{*} \cdot \widehat{\boldsymbol{\sigma}}\right)\left(\mathbf{v}_{21}^{*} \cdot \widehat{\boldsymbol{\sigma}}\right)^{3}, \\
\xi_{0}=-4 h n \bar{\sigma}^{2} v_{f} \frac{1}{\bar{\phi}} \int d \mathbf{v}_{0}^{*} d \mathbf{v}_{1}^{*} \int d \Omega f_{0}^{(2) *}\left(\mathbf{v}_{0}^{*}, \mathbf{v}_{1}^{*}, \mathbf{r}_{10}=-\overline{\boldsymbol{\sigma}}\right) \Theta\left(\mathbf{v}_{10}^{*} \cdot \widehat{\boldsymbol{\sigma}}\right)\left(\mathbf{v}_{10}^{*} \cdot \widehat{\boldsymbol{\sigma}}\right)^{2} \\
\times\left(h \mathbf{v}_{10}^{*}+\mathbf{v}_{0}^{*}\right) \cdot \widehat{\boldsymbol{\sigma}} \\
\nu_{0}=-2 h n \bar{\sigma}^{2} v_{f} \frac{1}{\bar{\phi}} \int d \mathbf{v}_{0}^{*} d \mathbf{v}_{1}^{*} \int d \Omega f_{0}^{(2) *}\left(\mathbf{v}_{0}^{*}, \mathbf{v}_{1}^{*}, \mathbf{r}_{10}=-\overline{\boldsymbol{\sigma}}\right) \Theta\left(\mathbf{v}_{10}^{*} \cdot \widehat{\boldsymbol{\sigma}}\right)\left(\mathbf{v}_{10}^{*} \cdot \widehat{\boldsymbol{\sigma}}\right)^{2} \mathbf{v}_{0}^{*} \cdot \widehat{\boldsymbol{\sigma}} .
\end{aligned}
$$

All velocities have been scaled relative to $v_{f}=\sqrt{\left\langle v^{2}\right\rangle}$ and

$$
f^{(2)}=v_{f}^{-6} f^{(2) *}, \quad f_{0}^{(2)}=v_{f}^{-6} f_{0}^{(2) *} .
$$

The results at this point are still exact. It follows directly from these results that $\xi$ and $\omega_{D}$ are manifestly positive.

\section{Neglect of velocity correlations}

If velocity correlations in the reduced distribution functions are neglected on the precollision hemispheres [15, 16$]$ they simplify to

$$
f^{(2) *}\left(\mathbf{v}_{1}^{*}, \mathbf{v}_{2}^{*}, \mathbf{r}_{21}=-\boldsymbol{\sigma}\right)=g f^{*}\left(v_{1}^{*}\right) f^{*}\left(v_{2}^{*}\right), \quad f_{0}^{(2) *}\left(\mathbf{v}_{0}^{*}, \mathbf{v}_{1}^{*}, \mathbf{r}_{10}=-\overline{\boldsymbol{\sigma}}\right)=g_{0} f_{0}^{*}\left(v_{0}^{*}\right) f^{*}\left(v_{1}^{*}\right),
$$

where $g$ and $g_{0}$ are the fluid-fluid and fluid-impurity pair correlation functions for particles at contact. The angular integrals can now be performed to give

$$
\begin{gathered}
\xi=\frac{1}{8} n \pi \sigma^{2} v_{f} g\left(1-\alpha^{2}\right) \int d \mathbf{v}_{1}^{*} d \mathbf{v}_{2}^{*} f^{*}\left(v_{1}^{*}\right) f^{*}\left(v_{2}^{*}\right) v_{21}^{* 3}, \\
\xi_{0}=\frac{8 \pi\left\langle v^{*}\right\rangle}{3} h n \bar{\sigma}^{2} v_{f} g_{0} \frac{1}{\bar{\phi}}\left[\left\langle v_{0}^{* 2} \gamma^{*}\left(v_{0}^{* 2}\right)\right\rangle-h\left\langle n_{1}^{*}\left(v_{0}^{* 2}\right)\right\rangle\right], \\
\nu_{0}=\frac{4 \pi}{3}\left\langle v^{*}\right\rangle h n \bar{\sigma}^{2} v_{f} g_{0} \frac{1}{\bar{\phi}}\left\langle v_{0}^{* 2} \gamma^{*}\left(v_{0}^{* 2}\right)\right\rangle,
\end{gathered}
$$

where the dimensionless functions $\gamma^{*}\left(v_{0}^{* 2}\right)$ and $n_{1}^{*}\left(v_{0}^{* 2}\right)$ have been introduced for connection with the discussion in Appendix B,

$$
\gamma^{*}\left(v_{0}^{* 2}\right)=\frac{3}{4 v_{0}^{*}\left\langle v^{*}\right\rangle} \int d \mathbf{v}_{1}^{*} f^{*}\left(v_{1}^{*}\right) v_{01}^{*} \widehat{\mathbf{v}}_{0} \cdot \mathbf{v}_{01}^{*}, \quad n_{1}^{*}\left(v_{0}^{* 2}\right)=\frac{3}{4\left\langle v^{*}\right\rangle} \int d \mathbf{v}_{1}^{*} f^{*}\left(v_{1}^{*}\right) v_{10}^{* 3}
$$

\section{Maximum entropy ensemble}

The HCS distributions are not known exactly, although approximate evaluations suggest they are close to Maxwellians. Therefore, to obtain an estimate for the cooling rates and collision frequency the maximum entropy (information) ensemble is assumed in this section. This is the Gaussian whose density, momentum, and kinetic energy are constrained to have the same values as for the HCS,

$$
f^{*}\left(v^{*}\right)=\left(\frac{3}{2 \pi}\right)^{3 / 2} e^{-3 v^{* 2} / 2}, \quad f_{0}^{*}\left(v_{0}^{*}\right)=\left(\frac{3}{2 \pi \bar{\phi}}\right)^{3 / 2} e^{-3 v_{0}^{* 2} / 2 \bar{\phi}} .
$$


This gives the results

$$
\begin{gathered}
\xi=\frac{4}{3} \sqrt{\frac{\pi}{3}} n \sigma^{2} v_{f} g\left(1-\alpha^{2}\right), \quad \xi_{0}=2 \nu_{0}\left(1-h \frac{1+\bar{\phi}}{\bar{\phi}}\right) \\
\nu_{0}=\frac{8}{3} \sqrt{\frac{2 \pi}{3}} h n \bar{\sigma}^{2} v_{f} g_{0}(1+\bar{\phi})^{1 / 2} .
\end{gathered}
$$

\section{APPENDIX B: ASYMPTOTIC KINETIC EQUATIONS}

The analysis here is based on the Enskog-Lorentz equation to describe the distribution function for the impurity particle. Interest is restricted to the case of small ratio of fluid particle mass to impurity particle mass. To obtain an asymptotic form for the kinetic equation, first a Kramers-Moyal expansion is performed to second order in the mass ratio. This accounts for the dependence of the collisional changes on the mass ratio. Subsequently, two different expansions are performed for the final asymptotic form depending on the value of a control parameter $\xi^{*}$.

\section{Kramers-Moyal Expansion}

The Kramers-Moyal expansion of the Enskog-Lorentz equation has been obtained in Appendix A of reference [3]. The result is

$$
\begin{aligned}
\partial_{t} f_{0}\left(\mathbf{v}_{0}, t\right)= & \frac{\partial}{\partial \mathbf{v}_{0}} \cdot\left[h \mathbf{v}_{0} \gamma\left(v_{0}\right) f_{0}\left(\mathbf{v}_{0}, t\right)\right]+\frac{1}{2} \frac{\partial^{2}}{\partial v_{0 i} \partial v_{0 j}}\left\{h ^ { 2 } \left[n_{1}\left(v_{0}\right) \delta_{i j}\right.\right. \\
& \left.\left.+n_{2}\left(v_{0}\right)\left(v_{0 i} v_{0 j}-\frac{1}{3} \delta_{i j} v_{0}^{2}\right)\right] f_{0}\left(\mathbf{v}_{0}, t\right)\right\}+\mathcal{O}\left(h^{3}\right)
\end{aligned}
$$

The friction $\gamma\left(v_{0}\right)$ and noise $n_{1}\left(v_{0}\right), n_{2}\left(v_{0}\right)$ are

$$
\gamma\left(v_{0}\right)=\frac{\nu_{c}}{2 h} \gamma^{*}(\phi), \quad n_{1}\left(v_{0}\right)=\frac{\nu_{c}}{3 h} v_{f}^{2} n_{1}^{*}(\phi), \quad n_{2}\left(v_{0}\right)=\frac{3 \nu_{c}}{5 h} n_{2}^{*}(\phi)
$$

where $\nu_{c}=\frac{8}{3} h n \pi \bar{\sigma}^{2} g_{0}\langle v\rangle$ is the characteristic impurity collision frequency introduced in $(3)$. Also $\gamma^{*}(\phi)$ and $n_{1}^{*}(\phi)$ have been defined in (A27), and

$$
n_{2}^{*}(\phi)=\frac{15}{16\left\langle v^{*}\right\rangle \phi} \int d \mathbf{v}_{1}^{*} f^{*}\left(v_{1}^{*}\right) v_{01}^{*}\left[\left(\mathbf{v}_{01}^{*} \cdot \widehat{\mathbf{v}}_{0}\right)^{2}-\frac{1}{3} v_{01}^{* 2}\right] .
$$

The dimensionless variables are

$$
\phi=v_{0}^{* 2}, \quad v_{0}^{*}=v_{0} / v_{f}, \quad \mathbf{v}_{1}^{*}=\mathbf{v}_{1} / v_{f}, \quad \mathbf{v}_{01}^{*}=\phi^{1 / 2} \widehat{\mathbf{v}}_{0}-\mathbf{v}_{1}^{*}, \quad v_{f}=\sqrt{\left\langle v^{2}(t)\right\rangle} .
$$

The analysis of the deterministic limit in the text makes use of the property $\gamma^{*}(\phi) \geq \gamma^{*}(0)=1$. To prove this, first perform the angle integrations to get

$$
\begin{aligned}
\gamma^{*}(\phi) & =1+\frac{1}{5\left\langle v^{*}\right\rangle}\left[\phi\left\langle v^{*-1}\right\rangle-\pi \phi \int_{0}^{\sqrt{\phi}} d v^{*} f^{*}\left(v^{*}\right) v^{*}\left(4+\frac{v^{*}}{\sqrt{\phi}}\right)\left(1-\frac{v^{*}}{\sqrt{\phi}}\right)^{4}\right] \\
& \geq 1+\frac{1}{5\left\langle v^{*}\right\rangle}\left[\phi\left\langle v^{*-1}\right\rangle-4 \pi \phi \int_{0}^{\sqrt{\phi}} d v^{*} f^{*}\left(v^{*}\right) v^{*}\right],
\end{aligned}
$$

where the inequality results from $4 \geq(4+x)(1-x)^{4}$ for $x \leq 1$. Next, writing out the contribution from $\left\langle v^{*-1}\right\rangle$ explicitly gives the desired result

$$
\gamma^{*}(\phi) \geq 1+\frac{4 \pi \phi}{5\left\langle v^{*}\right\rangle} \int_{\sqrt{\phi}}^{\infty} d v^{*} f^{*}\left(v^{*}\right) v^{*} \geq \gamma^{*}(0)=1 .
$$


Analogously, it is possible to prove that $\gamma^{* \prime}(\phi) \geq 0$ :

$$
\begin{aligned}
\gamma^{* \prime}(\phi) & =\frac{1}{5\left\langle v^{*}\right\rangle}\left[\left\langle v^{*-1}\right\rangle-\frac{\pi}{2} \int_{0}^{\sqrt{\phi}} d v^{*} f^{*}\left(v^{*}\right) v^{*}\left(8+9 \frac{v^{*}}{\sqrt{\phi}}+3 \frac{v^{* 2}}{\phi}\right)\left(1-\frac{v^{*}}{\sqrt{\phi}}\right)^{3}\right] \\
& \geq \frac{1}{5\left\langle v^{*}\right\rangle}\left[\left\langle v^{*-1}\right\rangle-4 \pi \int_{0}^{\sqrt{\phi}} d v^{*} f^{*}\left(v^{*}\right) v^{*}\right] \\
& =\frac{4 \pi}{5\left\langle v^{*}\right\rangle} \int_{\sqrt{\phi}}^{\infty} d v^{*} f^{*}\left(v^{*}\right) v^{*} \geq 0 .
\end{aligned}
$$

The remaining analysis of the text and below does not require the explicit forms for $\gamma^{*}(\phi), n_{1}^{*}(\phi)$, and $n_{2}^{*}(\phi)$. However, for the illustrations in the graphs an excellent approximation is obtained using the maximum entropy ensemble (A28) for the fluid; no assumption is required regarding the impurity particle distribution. The resulting integrals can be performed with the results

$$
\begin{gathered}
\gamma^{*}(\phi)=\frac{1}{8 \phi}(1+3 \phi) e^{-3 \phi / 2}-\frac{1}{16 \phi^{3 / 2}} \sqrt{\frac{2 \pi}{3}}\left(1-6 \phi-9 \phi^{2}\right) \operatorname{erf}(\sqrt{3 \phi / 2}), \\
n_{1}^{*}(\phi)=\frac{1}{8}(5+3 \phi) e^{-3 \phi / 2}+\frac{1}{16} \sqrt{\frac{2 \pi}{3 \phi}}\left(3+18 \phi+9 \phi^{2}\right) \operatorname{erf}(\sqrt{3 \phi / 2}), \\
n_{2}^{*}(\phi)=\frac{5}{48 \phi^{2}}\left(-1+2 \phi+3 \phi^{2}\right) e^{-3 \phi / 2}+\frac{5}{96 \phi^{5 / 2}} \sqrt{\frac{2 \pi}{3}}\left(1-3 \phi+9 \phi^{2}+9 \phi^{3}\right) \operatorname{erf}(\sqrt{3 \phi / 2}) .
\end{gathered}
$$

In the following only solutions that depend on the magnitude of $\mathbf{v}_{0}$ are considered. Since the order parameter is the average of $\phi$,

$$
\bar{\phi}(t)=\int d \mathbf{v}_{0} v_{0}^{* 2} f_{0}\left(v_{0}, t\right)
$$

it is appropriate to change variables from $v_{0}$ to $\phi$. In addition, the dimensionless time scale of (5) is introduced. This is accomplished by defining the new distribution function $P(\phi, s)$ by

$$
P(\phi, s) \equiv 4 \pi f_{0}\left(v_{0}, t\right) v_{0}^{2} \frac{d v_{0}}{d \phi}=2 \pi v_{f}^{3} \phi^{1 / 2} f_{0}\left(v_{0}, t\right),
$$

or

$$
f_{0}\left(v_{0}, t\right)=\frac{1}{2 \pi v_{f}^{3}} \phi^{-1 / 2} P(\phi, s)
$$

The Kramers-Moyal equation (B1) becomes for $P(\phi, s)$

$$
\begin{aligned}
\partial_{s} P(\phi, s)= & \frac{\partial}{\partial \phi}\left\{\phi\left[-\xi^{*}+\gamma^{*}(\phi)\right]-\left(1-\frac{2}{3} \frac{\partial}{\partial \phi} \phi\right) h n_{1}^{*}(\phi)+\frac{4}{5} \frac{\partial}{\partial \phi} \phi^{2} h n_{2}^{*}(\phi)\right\} P(\phi, s) \\
& +\mathcal{O}\left(h^{2}\right) .
\end{aligned}
$$

The deterministic limit, $h=0$, is described in the text. In the following an outline of the fluctuations about this determistic limit is given.

\section{Expansion around $\bar{\phi}_{0 \mathrm{~s}}$}

The effects of finite $h$ represent "noise" which broadens the width of the initial delta function as the system evolves. To include such effects consider solutions of the form 


$$
P(\phi, s, h)=h^{-p} \mathcal{P}\left(\frac{\phi-\bar{\phi}_{0 \mathrm{~s}}}{h^{p}}, s, h\right),
$$

such that the $\operatorname{limit}_{h \rightarrow 0} \mathcal{P}(\eta, s, h)=\mathcal{P}(\eta, s)$ is finite and independent of $h$. The choice of reference state $\bar{\phi}_{0 \text { s }}$ given by (51) implies initial conditions that do not deviate too much from the stationary state. To find such solutions, define a change of variables in (B14) by

$$
\phi=\bar{\phi}_{0 \mathrm{~s}}+h^{p} \eta, \quad P(\phi, s, h)=h^{-p} \mathcal{P}(\eta, s, h) .
$$

In the ordered phase a non-trivial equation for $\mathcal{P}$ is obtained with the choice $p=1 / 2$,

$$
\partial_{s} \mathcal{P}(\eta, s, 0) \rightarrow \frac{\partial}{\partial \eta}\left\{\eta \bar{\phi}_{0 \mathrm{~s}} \gamma^{* \prime}\left(\bar{\phi}_{0 \mathrm{~s}}\right)+\bar{\phi}_{0 \mathrm{~s}}\left[\frac{2}{3} n_{1}^{*}\left(\bar{\phi}_{0 \mathrm{~s}}\right)+\frac{4}{5} \bar{\phi}_{0 \mathrm{~s}} n_{2}^{*}\left(\bar{\phi}_{0 \mathrm{~s}}\right)\right] \frac{\partial}{\partial \eta}\right\} \mathcal{P}(\eta, s, 0), \quad \xi^{*}>1 .
$$

where it has been recognized that $\xi^{*}-\gamma^{*}\left(\bar{\phi}_{\mathrm{s}}\right)=0$ in this phase. The average value of $\eta$ obeys the equation

$$
\partial_{s} \bar{\eta}(s)=-\bar{\phi}_{0 \mathrm{~s}} \gamma^{* \prime}\left(\bar{\phi}_{0 \mathrm{~s}}\right) \bar{\eta}(s) \text {, }
$$

which is the linearized form of the deterministic dynamics (50) for $\xi^{*}>1$. Stability is assured by $\gamma^{* \prime}\left(\bar{\phi}_{0 \mathrm{~s}}\right) \geq 0$, which is seen to be the case using $(\overline{\mathrm{B} 7})$. The stationary solution to $(\overline{\mathrm{B} 18})$ is $\bar{\eta}_{\mathrm{s}}=0$ so there are no corrections to $\bar{\phi}_{0 \mathrm{~s}}$ in this limit. The stationary solution for the distribution function is obtained from

$$
\left[\frac{2}{3} n_{1}^{*}\left(\bar{\phi}_{0 \mathrm{~s}}\right)+\frac{4}{5} \bar{\phi}_{0 \mathrm{~s}} n_{2}^{*}\left(\bar{\phi}_{0 \mathrm{~s}}\right)\right] \frac{\partial}{\partial \eta} \mathcal{P}_{\mathrm{s}}=-\bar{\phi}_{0 \mathrm{~s}} \gamma^{* \prime}\left(\bar{\phi}_{0 \mathrm{~s}}\right) \eta \mathcal{P}_{\mathrm{s}}
$$

whose solution is

$$
\mathcal{P}_{\mathrm{s}}(\eta)=\frac{1}{\sqrt{2 B\left(\bar{\phi}_{0 \mathrm{~s}}\right) \pi}} e^{-\eta^{2} / 2 B\left(\bar{\phi}_{0 \mathrm{~s}}\right)}, \quad B\left(\bar{\phi}_{0 \mathrm{~s}}\right)=\frac{1}{\gamma^{* \prime}\left(\bar{\phi}_{0 \mathrm{~s}}\right)}\left[\frac{2}{3} n_{1}^{*}\left(\bar{\phi}_{0 \mathrm{~s}}\right)+\frac{4}{5} \bar{\phi}_{0 \mathrm{~s}} n_{2}^{*}\left(\bar{\phi}_{0 \mathrm{~s}}\right)\right],
$$

or, in terms of $\phi$,

$$
P_{\mathrm{s}}(\phi)=\frac{1}{\sqrt{2 B\left(\bar{\phi}_{0 \mathrm{~s}}\right) h \pi}} e^{-\left(\phi-\bar{\phi}_{0 \mathrm{~s}}\right)^{2} / 2 B\left(\bar{\phi}_{0 \mathrm{~s}}\right) h} .
$$

In the normal phase $\bar{\phi}_{0 \mathrm{~s}}=0$ and the change of variables in (B16) becomes $\phi=h^{p} \eta$. A non-trivial equation for $\mathcal{P}$ is obtained with $p=1$,

$$
\partial_{s} \mathcal{P}(\eta, s, 0) \rightarrow \frac{\partial}{\partial \eta}\left[\left(1-\xi^{*}\right) \eta-\left(1-\frac{2}{3} \frac{\partial}{\partial \eta} \eta\right) n_{1}^{*}(0)\right] \mathcal{P}(\eta, s, 0), \quad \xi^{*}<1 .
$$

The average value of $\eta$ now obeys the equation

$$
\left[\partial_{s}+\left(1-\xi^{*}\right)\right] \bar{\eta}(s)=n_{1}^{*}(0)
$$

The stationary solution to this equation is

$$
\bar{\eta}_{\mathrm{s}}=\frac{n_{1}^{*}(0)}{1-\xi^{*}}
$$

which gives the leading finite contribution to $\bar{\phi}_{\mathrm{s}}$ as $h \rightarrow 0$. The stationary distribution function is

$$
\mathcal{P}_{\mathrm{s}}(\eta)=\frac{3}{\bar{\eta}_{\mathrm{s}}}\left(\frac{3 \eta}{2 \bar{\eta}_{\mathrm{s}} \pi}\right)^{1 / 2} e^{-3 \eta / 2 \bar{\eta}_{\mathrm{s}}}
$$

and the corresponding distribution in terms of $\phi$ is

$$
P_{\mathrm{s}}(\phi)=\frac{3}{h \bar{\eta}_{\mathrm{s}}}\left(\frac{3 \phi}{2 h \bar{\eta}_{\mathrm{s}} \pi}\right)^{1 / 2} e^{-3 \phi / 2 h \bar{\eta}_{\mathrm{s}}}
$$


* Permanent address: Departamento de Física, Universidad de Extremadura, E-06071 Badajoz, Spain; Email address: andres@unex.es

$\dagger \quad$ Email address: dufty@phys.ufl.edu

[1] V. Garzó and J. Dufty, Phys. Rev. E 60, 5706 (1999).

[2] J. M. Montanero and V. Garzó, Gran. Matt. (submitted).

[3] J. J. Brey, J. W. Dufty, and A. Santos, J. Stat. Phys. 97, 2811 (1999).

[4] J. J. Brey, M. J. Ruiz-Montero, R. García-Rojo, and J. W. Dufty, Phys. Rev. E 60, 7174 (1999).

[5] A. Santos and J. W. Dufty, Phys. Rev. Lett. 86, 4823 (2001).

[6] J. W. Dufty and V. Garzó, J. Stat. Phys. (submitted).

[7] P. A. Martin and J. Piasecki, Europhys. Lett. 46, 613 (1999).

[8] Following the thermodynamic analogy, the critical exponents $\hat{\delta}, \hat{\beta}, \hat{\gamma}$, and $\hat{\alpha}$ are defined as $\bar{\phi}_{\mathrm{s}}\left(\xi^{*}=1, h\right) \sim h^{1 / \hat{\delta}}, \bar{\phi}_{\mathrm{s}}\left(\xi^{*}>\right.$ $1, h=0) \sim\left(\xi^{*}-1\right)^{\hat{\beta}}, \chi\left(\xi^{*}, h=0\right) \sim\left|\xi^{*}-1\right|^{-\hat{\gamma}}, C_{h}\left(\xi^{*}, h=0\right) \sim\left|\xi^{*}-1\right|^{-\hat{\alpha}}$

[9] J. W. Dufty, J. Phys.: Condens. Matter 12, 47 (2000).

[10] J. W. Dufty, J. J. Brey, and J. F. Lutsko (unpublished).

[11] N. van Kampen, Stochastic Processes in Physics and Chemisty (North-Holland, New York, 1981).

[12] Note that the friction coefficient $\bar{\gamma}^{*}(\bar{\phi})=(1+\bar{\phi})^{1 / 2}$ and noise coefficient $\bar{n}(\bar{\phi})=(1+\bar{\phi})^{3 / 2}$ introduced in the phenomenological description of Section II are $\bar{\gamma}^{*}(\bar{\phi})=\bar{\phi}^{-1}\left\langle\phi \gamma^{*}(\phi)\right\rangle$ and $\bar{n}(\bar{\phi})=\left\langle n_{1}^{*}(\phi)\right\rangle$, where the averages are taken with the maximum entropy ensemble.

[13] V. Zubov, Methods of Lyapunov and their Application (P. Noordhoff Ltd., Groningen, 1964).

[14] J. J. Brey, J. W. Dufty, and A. Santos, J. Stat. Phys. 87, 1051 (1997).

[15] J. F. Lutsko, Phys. Rev. Lett. 86, 3344 (2001); Phys. Rev. E 63, 061211 (2001).

[16] R. Soto and M. Mareschal, Phys. Rev. E 63, 041303 (2001). 